Pacific Journal of Mathematics

STRUCTURE THEORY FOR A CLASS OF CONVOLUTION 


\section{STRUCTURE THEORY FOR A CLASS OF CONVOLUTION ALGEBRAS}

\section{Edwin HewitT and Herbert S. Zuckerman}

Introduction. This paper is a chapter in the study of convolution algebras begun in [7]. The algebras studied here are algebras of Borel measures on certain compact semigroups, and we describe completely the structure of these algebras. The solution obtained seems remarkable in view of the extreme complexity of the corresponding measure algebras for compact Abelian groups (see [12]). Our success is explained by the simple algebraic structure of the semigroups we deal with.

In addition to the structure theory $(\S \S 2-6)$, we give an application to probability $(\S 7)$, and some concrete examples and illustrations $(\S 8)$.

Throughout this paper, we use the notation and terminology of [7]. In particular, the reader should be familiar with $\S 1$ of [7]. The related papers [6] and [8] are not essential for understanding the present paper, but are referred to occasionally here at points of contact in subjectmatter. For all measure-theoretic terms and techniques not explained here, see [4]. References are made throughout the present paper to [9] for topological matters, and to [10] for the elementary theory of Banach algebras. We use $K$ to denote the complex number system. All other special symbols will be explained as they appear.

\section{The semigroups to be studied.}

1.1. We consider an arbitrary non-void set $G$, completely ordered by a transitive, irreflexive relation " $<$ ". That is, for all $x, y \in G$, exactly one of the relations $x<y, x=y, y<x$ obtains, and the relations $x<y$ and $y<z$ imply $x<z$. As usual, we write $y>x$, meaning $x<y$, and we write $x \leqq y$, meaning $x<y$ or $x=y$. For $u, v \in G$, we define

$$
\begin{array}{ll}
] u, v[=\{x: x \in G, u<x<v\} & \text { (open interval), } \\
{[u, v[=\{x: x \in G, u \leqq x<v\}} & \text { (half-open interval), } \\
] u, v]=\{x: x \in G, u<x \leqq v\} & \text { (half-open interval), } \\
{[u, v]=\{x: x \in G, u \leqq x \leqq v\}} & \text { (closed interval) }
\end{array}
$$

These sets may or may not be void, depending upon the relation between $u$ and $v$.

Received January 17, 1956. The first-named author is a fellow of the John Simon Guggenheim Memorial Foundation. Research also supported in part by the National Science Foundation. 
1.2. We make $G$ into a semigroup by defining the product $x y$ as $\max (x, y)$ for all $x, y \in G$. It is obvious that $x(y z)=(x y) z$ for all $x, y$, $z \in G$, that $x y=y x$ for all $x, y \in G$, and that $x^{2}=x$ for all $x \in G$.

1.3. Being a completely ordered set, $G$ has a natural topology defined in terms of the ordering. For all $a \in G$ and all $u, v \in G$ such that $u<$ $a<v$, the open interval $] u, v[$ is taken as an open neighborhood of the point $a$. If there is no $u$ such that $u<a$ (i.e., if $a$ is the first element of $G$ ), then [a, $v$ [ with $v>a$ is a neighborhood of $a$, and analogously if $a$ is the last element of $G$. These are all of the open neighborhoods of $a$. It is obvious that Hausdorff's neighborhood axioms are satisfied and that Hausdorff's separation axiom is satisfied. A point $a$ in $G$ is isolated if and only if it has an immediate predecessor and an immediate successor. It has a complete neighborhood system consisting of intervals $[a, v[$ (]$u, a])$ if and only if it has an immediate predecessor (an immediate successor).

It is easy to verify that the semigroup operation $x y=\max (x, y)$ is continuous in both $x$ and $y$ for the topology described above. Hence $G$ is a topological semigroup satisfying the Hausdorff separation axiom.

1.4. We impose the additional restriction on $G$ that it be compact in the interval topology $y^{1}$. For this, it is both necessary and sufficient that every subset of $G$ admit a least upper bound and a greatest lower bound. In particular, $G$ has a least element, which we shall call $\alpha$, and a greatest element, which we shall call $\omega$ (not to be confused with the ordinal number $\omega$ ). For a sketch of the proof of this, see [9], p. 162, exercise $C$.

1.5. From now on, we shall suppose, save where the contrary is explicitly stated, that $G$ is a completely ordered set that is compact in the interval topology, and made into a topological semigroup by the operation $\max (x, y)$.

1.6. Let $\mathfrak{S}(G)$ denote the linear space of all complex-valued continuous functions on $G$. We give $\mathfrak{S}(G)$ the usual norm:

$$
\|f\|=\max _{x \in G}|f(x)|
$$

for $f \in \mathfrak{S}(G)$. Let $\tilde{\mathfrak{S}}(G)$ denote the conjugate space of $\mathfrak{C}(G)$, that is, the linear space of all complex-valued linear functionals $L$ on $\mathfrak{S}(G)$ such that the number

$$
\|L\|=\sup \{|L(f)|: f \in \mathbb{\subseteq}(G),\|f\| \leqq 1\}
$$

is finite. It is well known that each $L \in \widetilde{\mathfrak{S}}(G)$ has a unique representation

1 See however 8.5. 
as an integral with respect to a complex-valued, countably additive, regular measure $\lambda$ defined on all Borel subsets of $G$ (see [4], pp. 247-248). That is,

$$
L(f)=\int_{G} f(x) d \lambda(x)
$$

for all $f \in \mathfrak{S}(G)$. While many authors have contributed to this theorem, we call it for convenience the Riesz representation theorem. Elements of $\widetilde{\mathfrak{S}}(G)$ will be denoted by capital Roman letters, $L, M, \cdots$, and the corresponding measures of the kind referred to will be denoted by the corresponding lower-case Greek letters $\lambda, \mu, \cdots$. Under our interpretation of the term "measure," the measures $\lambda, \mu, \cdots$ are set-functitns and not linear functionals (for a different point of view, consult [2], passim). However, we shall allow ourselves the abuse of notation $\lambda \in \widetilde{\mathcal{C}}(G)$, meaning that $\lambda$ is connected with an element $L \in \widetilde{\mathfrak{S}}(G)$ by the relation 1.6.1. At various points in our discussion, it will be necessary to pass from an element $L \in \widetilde{\mathscr{C}}(G)$ to the corresponding measure $\lambda$. For nonnegative $L$ (that is, $L(f) \geqq 0$ for $f$ real and nonnegative), this process is simple. Let $F$ be any closed subset of $G$. Then

$$
\begin{gathered}
\lambda(F)=\inf \{L(f): f \in \mathfrak{C}(G), f(x) \geqq 1 \text { for } x \in F, \\
f(x) \geqq 0 \text { for } x \in G\} .
\end{gathered}
$$

Let $H$ be any open subset of $G$. Then

$$
\lambda(H)=\sup \{\lambda(F): F \text { is closed, } F \subset H\} .
$$

Let $X$ be any subset of $G$. Then

$$
\lambda(X)=\inf \{\lambda(H): H \text { is open, } H \supset X\} \text {. }
$$

These three definitions of $\lambda$, on various families of sets, are all consistent, and $\lambda$ is an outer measure on all subsets of $G$. Every Borel set is $\lambda$ measurable, $\lambda$ is regular, and 1.6.1 holds.

For an arbitrary $L \in \widetilde{\mathfrak{S}}(G)$, we obtain the corresponding measure $\lambda$ by writing $L$ as

$$
L=L_{1}-L_{2}+i\left(L_{3}-L_{4}\right),
$$

where $L_{1}, \cdots, L_{4}$ are non-negative functionals on $\mathbb{S}(G)$.

1.7. We recall that a semicharacter of a semigroup $H$ is a bounded complex-valued function $\chi$ on $H$, not identically zero, satisfying the functional equation $\chi(x y)=\chi(x) \chi(y)$ for all $x, y \in H$ ([7], 3.1 and [8], 1.3). Semicharacters of our semigroup $G$ play a vital rôle in the solution of the present problem, and we proceed to identify the semicharacters of $G$. 
1.8. THEOREM. Let $G$ be as specified in 1.5. Then functions of the following two types are semicharacters of $G$ :

functions $\psi_{a]}$, where $a \in G$ and

$$
\psi_{a]}(x)=\left\{\begin{array}{l}
1 \text { if } x \leqq a \\
0 \text { if } x>a
\end{array}\right.
$$

functions $\psi_{a[}$, where $\left.\left.a \in\right] \alpha, \omega\right]$ and

$$
\psi_{a_{\mathrm{I}}}(x)=\left\{\begin{array}{ll}
1 & \text { if } x<a, \\
0 & \text { if } x \geqq a
\end{array} .\right.
$$

Furthermore, every semicharacter $\chi$ of $G$ is one of these two types.

Proof. It is easy to see that all functions $\psi_{a 1}^{\prime}(a \in G)$ and $\psi_{a \mid}(\alpha<$ $a \leqq \omega)$ are semicharacters, and we omit the verification. To establish the converse, let $\chi$ be a semicharacter of $G$. Since $x^{2}=x$ for all $x \in G$, $\chi$ assumes no values other than 0 and 1 . If $\chi$ is identically 1 (in this case we write $\chi=1)$, then $\chi=\psi_{\omega]}$. If $\chi \neq 1$, then there exist $a$ and $b$ such that $\chi(a)=1, \chi(b)=0$. Let $A=\{x ; x \in G, \chi(x)=1\}, B=\{x ; x \in G$, $\chi(x)=0\}$. If $x \in A$ and $x^{\prime}<x$, then we have $1=\chi(x)=\chi\left(x^{\prime} x\right)=\chi\left(x^{\prime}\right) \chi(x)$ $=\chi\left(x^{\prime}\right)$. If $x \in B$ and $x^{\prime}>x$, then we have $\chi\left(x^{\prime}\right)=\chi\left(x^{\prime} x\right)=\chi\left(x^{\prime}\right) \chi(x)=0$. The sets $A$ and $B$ are therefore non-void complementary sets forming a Dedekind cut in $G$. Since $G$ is compact, $A$ has a least upper bound $a$. If $a \in A$, we have $\chi=\psi_{a]}$; if $a \in B$, we have $\chi=\psi_{a \text { [ }}$.

1.9. Theorem. Let $G$ be as specified in 1.5. Suppose first that $\alpha \leqq a<\omega$. Then the function $\psi_{a]}$ (1.8.1) is continuous if and only if $a$ has an immediate successor. The function $\psi_{\omega]}$ is trivially continuous. Suppose next that $\alpha<a \leqq \omega$. Then the function $\psi_{a[}$ is continuous if and only if $a$ has an immediate predecessor $a_{-}$, and in this case, $\psi_{a_{[}}=\psi_{\left.a_{-}\right]}$.

We omit the proof of this theorem.

1.10. THEOREM. The semigroup $G$ admits a continuous semicharacter different from 1 if and only if $G$ is disconnected.

Proof. Since a semicharacter of $G$ can assume only the values 0 and 1 , the necessity of the condition is obvious. Conversely, suppose that $G$ is disconnected, and that $P$ and $Q$ are non-void complementary open sets in $G$. Since $\sup P \in P$ and $\sup Q \in Q(P$ and $Q$ being closed), we may suppose without loss of generality that $\sup P<\omega$. Let $B=$ $\{x ; x \in G, x>\sup P\}$. If $\sup P=\inf B$, then every open interval containing sup $P$ contains points of $B$, and $B \subset Q$. Since $P$ is open, this cannot occur. Hence $\sup P<\inf B$, and the function $\psi_{\text {sup } P]}$ is a continu- 
ous nonconstant semicharacter.

1.11. REMARK. If $G$ is not compact in its interval topology, then semicharacters of the types $1, \psi_{a]}$, and $\psi_{a[}$ may or may not exhaust the class of all semicharacters. If $G$ admits a Dedekind cut $\{A, B\}$ (where $A$ is the lower class) and where $A$ has no supremum and $B$ no infimum, then the function $\psi_{A}$ equal to 1 on $A$ and 0 on $B$ is a semicharacter different from $1, \psi_{a]}$, and $\psi_{a \text { I }}$ for any $a$. The proof of 1.8 shows that the existence of such a Dedekind cut is also necessary for the existence of a semicharacter different from 1 and all $\psi_{a]}$ and $\psi_{a[}$.

1.12. Theorem. Let $G$ be as in 1.5. Let $\hat{G}$ denote the set of all semicharacters of $G$. Then $\hat{G}$ is a semigroup under pointwise multiplication.

Proof. If $\chi_{1}$ and $\chi_{2}$ are semicharacters, then the product $\chi_{1} \chi_{2}$ $\left(\chi_{1} \chi_{2}(x)=\chi_{1}(x) \chi_{2}(x)\right.$ for $\left.x \in G\right)$ is obviously either 0 or a semicharacter. Since $\chi_{1}(\alpha)=\chi_{2}(\alpha)=1$, we cannot have $\chi_{1} \chi_{2}=0$.

2. The convolution algebra $\tilde{\widetilde{C}}(G)$. In a previous paper, we have introduced the general notion of a convolution algebra ([7], p. 69, 1.3). We shall show here that $\tilde{\mathfrak{C}}(G)$ is a convolution algebra, where $\mathfrak{F}=\mathfrak{C}(G)$.

2.1. Theorem. Let $x \in G$ and let $f \in \mathfrak{S}(G)$. Then the function ${ }_{x} f$ whose value at $y \in G$ is $f(x y)=f(\max (x, y))$ is continuous.

Proof. This assertion follows immediately from the fact that

$$
{ }_{x} f(y)=\left\{\begin{array}{l}
f(x) \text { for } y \leqq x, \\
f(y) \text { for } y>x
\end{array}\right.
$$

2.2. THEOREM. Let $f \in \mathbb{S}(G)$ and $L \in \widetilde{\mathbb{S}}(G)$. Then the function on $G$ whose value at $x \leqq G$ is $L\left({ }_{x} f\right)$ is continuous [we also write $L\left({ }_{x} f\right)$ as $\left.L_{y}(f(x y))\right]$.

Proof. Let $u, v \in G$ and suppose that $u \leqq v$. Then we have

$$
{ }_{v} f(y)-{ }_{u} f(y)= \begin{cases}f(v)-f(u) & \text { if } \quad \alpha \leqq y \leqq u, \\ f(v)-f(y) & \text { if } u \leqq y \leqq v, \\ 0 & \text { if } v \leqq y .\end{cases}
$$

Now let $\varepsilon$ be a positive real number, and let $x$ be an arbitrary element of $G$. Since $f$ is continuous, there exist $a, b \in G$ such that $a<x<b$ 
(we omit the obvious changes needed when $x=\alpha$ or $x=\omega$ ) and such that $|f(s)-f(t)|<\varepsilon \cdot\|L\|^{-1}$ for all $\left.s, t \in\right] a, b[$. It follows from 2.2.1 that $\left|x_{x^{\prime}} f(y)-{ }_{x} f(y)\right|<\varepsilon \cdot\|L\|^{-1}$ for all $\left.x^{\prime} \in\right] a, b[$ and all $y \in G$. Hence we have

$$
\left|L\left({ }_{x^{\prime}} f\right)-L\left({ }_{x} f\right)\right| \leqq\|L\| \cdot\left\|_{x^{\prime}} f-{ }_{x} f\right\|<\varepsilon .
$$

This completes the proof.

2.3. Theorem. Let $L$ and $M$ be elements of $\widetilde{\mathfrak{S}}(G)$. For all $f \in \mathfrak{C}(G)$, let $L * M(f)$ be the value assumed by the functional $L$ for the function whose value at $x$ is $M\left({ }_{x} f\right)$. We write

$$
L * M(f)=L_{x}\left(M_{y}(f(x y))\right) .
$$

Then $L * M \in \tilde{\mathbb{C}}(G)$, and

$$
\|L * M\| \leqq\|L\| \cdot\|M\|
$$

Proof. Theorem 2.2 shows that the right side of 2.3.1 has meaning. Now for all $x, y \in G$, we have $|f(x y)| \leqq\|f\|$, and hence $\|x\| \leqq\|f\|$. Therefore $\left|M_{y} f(x y)\right| \leqq\|M\| \cdot\|f\|$, and in turn $|L * M(f)| \leqq\|L\| \cdot\|M\| \cdot\|f\|$. This proves that $L^{*} M$ is a bounded functional, and since $L * M$ is obviously linear, 2.3.2 and the present theorem follow.

2.4. REMARK. Theorems 2.1, 2.2, and 2.3 are verifications of [7] 1.3.1, 1.3.2, and 1.3.3, respectively. Therefore we have proved that $\widetilde{\complement}(G)$ is a convolution algebra with the convolution $L * M$ of 2.3 .

2.5. Theorem. Let $L, M$ be elements of $\widetilde{\mathfrak{S}}(G)$ and let $\lambda, \mu$ be the corresponding measures as in 1.6. Then we have

$$
L * M(f)=\int_{G} \int_{G} f(\max (x, y)) d \mu(y) d \lambda(x),
$$

for all $f \in \mathfrak{S}(G)$.

Proof. The right side of 2.5.1 simply rewrites the right side of 2.3.1, making use of 1.6.1.

We shall write $\lambda * \mu$ to denote the measure associated with $L * M$ by 1.6.1.

2.6. TheOREM. The algebra $\widetilde{\Im}(G)$ is associative and commutative.

Proof. Associativity is a property of all convolution algebras ([7], p. 73, Theorem 1.5). Commutativity follows immediately from Fubini's theorem (which applies since all measures under consideration are finite and countably additive) and 2.5.1: 


$$
\begin{aligned}
L * M(f) & =\int_{G} \int_{G} f(\max (x, y)) d \mu(y) d \lambda(x)=\int_{G} \int_{G} f(\max (x, y)) d \lambda(x) d \mu(y) \\
& =\int_{G} \int_{G} f(\max (y, x)) d \lambda(x) d \mu(y)=M * L(f) .
\end{aligned}
$$

2.7. To identify the unit in $\widetilde{\mathfrak{S}}(G)$, and also for certain future purposes, we introduce a class of special linear functionals $E_{a}(a \in G)$ :

$$
E_{a}(f)=f(a) \text { for } f \in \mathfrak{S}(G) \text {. }
$$

It is clear that $\left\|t_{1} E_{a_{1}}+\cdots+t_{s} E_{a_{s}}\right\|=\sum_{j=1}^{s}\left|t_{j}\right|$ for all complex numbers $t_{1}$, $\cdots, t_{s}$ and distinct $a_{1}, \cdots, a_{s}$ in $G$. It is also clear that the measure $\varepsilon_{a}$ corresponding to $E_{a}$ is the unit mass at $a$ :

$$
\varepsilon_{a}(X)=\left\{\begin{array}{lll}
1 & \text { if } & a \in X \\
0 & \text { if } & a \notin X
\end{array}\right.
$$

for all $X \subset G$.

2.8. For all $\lambda \in \widetilde{\mathfrak{S}}(G)$ and every Borel set $A$ in $G$, let $\lambda^{A}$ be the measure such that $\lambda^{A}(X)=\lambda(A \cap X)$ for all Borel sets $X \subset G$.

2.9. Theorem. For all $\lambda \in \tilde{\mathbb{C}}(G)$ and all $a \in G$, we have

$$
\varepsilon_{a} * \lambda=\lambda([\alpha, a]) \varepsilon_{a}+\lambda^{j a, \omega]}
$$

and

$$
\varepsilon_{a} * \lambda=\lambda\left(\left[\alpha, a[) \varepsilon_{a}+\lambda^{[a, \omega]}\right.\right.
$$

Proof. The set $[\alpha, a]$ being a closed subset of $G$, it is certainly a Borel set (although not necessarily a Baire set), and hence $\lambda([\alpha, a])$ is defined. Similarly, ] $a, \omega]$ (which is void if $a=\omega$ ) is a Borel set, so that $\lambda^{[a, \omega]}$ is defined. Hence the right side of 2.9.1 is defined.

Consider the integral $I(x)=\int_{G} f(\max (x, y)) d \varepsilon_{a}(y)$, where $f \in \mathfrak{c}(G)$. The integrand has the constant value $f(x)$ for $y \in[\alpha, x]$, and is equal to $f$ in the interval $] x, \omega]$. Therefore if $x \leqq a$, then $I(x)=f(a)$. If $x>a$, then $I(x)=f(x)$. It follows that

$$
\begin{aligned}
L * E_{a}(f) & =\int_{G} I(x) d \lambda(x)=\int_{[\alpha, a]} f(a) \cdot 1 d \lambda(x)+\int_{1 a, \omega]} f(x) d \lambda(x) \\
& =\lambda([\alpha, a]) E_{a}(f)+\int_{G} f(x) d \lambda^{[a, \omega]}(x) .
\end{aligned}
$$

The relations 2.9.3 imply 2.9.1 immediately, and 2.9.2 is a trivial con- 
sequence of 2.9.1.

2.10. THEOREM. For all $\lambda \in \widetilde{\mathfrak{S}}(G)$, we have $\varepsilon_{\alpha} * \lambda=\lambda$. That is, $E_{\alpha}$ is the unit of $\tilde{\mathfrak{C}}(G)$.

Proof. Putting $a=\alpha$ in 2.9.1, aud taking an arbitrary Borel set $X \subset G$, we have

$$
\left.\left.\varepsilon_{\alpha} * \lambda(X)=\lambda(\{\alpha\}) \cdot \varepsilon_{\alpha}(X)+\lambda(] \alpha, \omega\right] \cap X\right) .
$$

If $\alpha \notin X$, then $\varepsilon_{\alpha}(X)=0$ and $\left.] \alpha, \omega\right] \cap X=X$. Hence $\varepsilon_{\alpha} * \lambda(X)=\lambda(X)$ in this case. If $\alpha \in X$, the right side of 2.10 .1 is equal to

$$
\lambda(\{\alpha\} \cap X)+\lambda(] \alpha, \omega] \cap X)=\lambda((\{\alpha\} \cap X) \cup(] \alpha, \omega] \cap X))=\lambda(X) .
$$

Therefore $\varepsilon_{\alpha} * \lambda(X)=\lambda(X)$ in all cases, and $\varepsilon_{\alpha} * \lambda=\lambda$.

2.11. THEOREM. For all $L \in \tilde{\mathfrak{S}}(G)$, we have $E_{\omega} * L=L(1) E_{\omega}$. In terms of measures, we have $\varepsilon_{\omega} * \lambda=\lambda([\alpha, \omega]) \varepsilon_{\omega}$.

Proof. The set $] \omega, \omega]$ is void, and so, putting $a=\omega$ in 2.9.1, we get $\lambda^{] \omega, \omega]}=0$ and $\varepsilon_{\omega} * \lambda=\lambda([\alpha, \omega]) \varepsilon_{\omega}$. The first statement is obviously equivalent to this.

2.12. TheOREM. For $a, b \in G$, we have $\varepsilon_{a} * \varepsilon_{b}=\varepsilon_{\max (a, b)}$.

Proof. This too follows at once from 2.9.1.

We summarize 2.3, 2.6, and 2.10 as follows.

2.13. THEOREM. Under the convolution 2.3.1, $\widetilde{\mathfrak{S}}(G)$ is a commutative Banach algebra with unit.

\section{The maximal ideals of $\tilde{\mathfrak{S}}(G)$.}

In this section, we identify all of the maximal ideals in $\tilde{\widetilde{C}}(G)$. Since $\widetilde{\mathfrak{G}}(G)$ is a commutative Banach algebra with unit, every maximal ideal in $\widetilde{\mathfrak{S}}(G)$ is closed and regular, and we may identify the class of maximal ideals in $\widetilde{\mathfrak{C}}(G)$ with the class of all (algebra) homomorphisms of $\widetilde{\mathfrak{S}}(G)$ onto $K$. For a discussion of Gel'fand's theory of commutative Banach algebras, see [10], pp. 66-81.

3.1. An obvious source of homomorphisms of $\widetilde{\mathfrak{S}}(G)$ onto $K$ is the 
set of all continuous semicharacters on $G$. If $\chi$ is such a semicharacter, then $L(\chi)$ is defined for all $L \in \widetilde{\mathbb{S}}(G)$ and the mapping

$$
L \rightarrow L(\chi)
$$

is obviously a linear functional on $\widetilde{\mathfrak{S}}(G)$. If $L, M \in \widetilde{\mathfrak{S}}(G)$, then

$$
\begin{aligned}
L * M(\chi) & =\int_{G} \int_{G} \chi(x y) d \mu(y) d \lambda(x)=\int_{G} \int_{G} \chi(x) \chi(y) d \mu(y) d \lambda(x) \\
& =\left[\int_{G} \chi(x) d \lambda(x)\right]\left[\int_{G} \chi(y) d \mu(y)\right]=L(\chi) M(\chi) .
\end{aligned}
$$

Hence the mapping 3.1.1 is multiplicative, that is, it is a homomorphism of $\tilde{\mathfrak{S}}(G)$ onto $K$.

However, as 1.8 and 1.10 show, $G$ may have very few continuous semicharacters. Indeed, it can be shown that there exist mappings of the form 3.1.1 carrying an arbitrary $L \neq 0$ into a non-zero number if and only if $G$ has Urysohn dimension zero. (We shall go no further into this minor point.) Therefore, if we have any hope of proving $\tilde{\mathfrak{S}}(G)$ semisimple, we must look further for homomorphisms of $\widetilde{\mathfrak{S}}(G)$ onto $K$. Our construction hinges on the fact that while the functions $\psi_{a 1}$ and $\psi_{a_{[}}$are often discontinuous, still they are Borel measurable and bounded. Therefore they are $\lambda$-integrable for all $\lambda \in \tilde{\widetilde{S}}(G)$ even though $L\left(\psi_{a]}\right)$ and $L\left(\psi_{a}\right)$ may be undefined $a b$ initio. The Riesz representation theorem gives us a canonical method of extending $L$ from $\mathcal{S}(G)$ to the space of all bounded Borel measurable functions on $G$, and it is just this fact that we use.

\subsection{Theorem. Let $a \in G$. Then the mapping}

$$
L \rightarrow \lambda([\alpha, \alpha])=\int_{G} \psi_{a]}(x) d \lambda(x) \quad(L \in \tilde{\mathbb{S}}(G))
$$

is a homomorphism of $\widetilde{\mathfrak{S}}(G)$ onto $K$. Let $a \in] \alpha, \omega]$. Then the mapping

$$
L \rightarrow \lambda\left(\left[\alpha, a[)=\int_{G} \psi_{a !}(x) d \lambda(x) \quad(L \in \widetilde{\widetilde{S}}(G))\right.\right.
$$

is a homomorphism of $\tilde{\mathbb{S}}(G)$ onto $K$.

Proof. First of all, it is clear that the mappings 3.2.1 and 3.2.2 are linear and not identically zero on $\tilde{\mathfrak{S}}(G)$. Our only task is to show that they are multiplicative. To this end, we consider first the mappings 3.2.1. If $a=\omega$, then we are dealing with the continuous semicharacter 1 , and this case has already been treated in 3.1. We may therefore suppose that $a<\omega$. If $a$ has an immediate successor $a_{+}$, then the interval $[\alpha, a]$ is open and closed, and the function $\psi_{a]}$ is a continuous 
semicharacter. Once again we can refer to 3.1. The remaining case is that in which $a<\omega$ and $a$ has no immediate successor. In this case, the interval $] a, u\left[\right.$ is non-void for every $u>a$, the semicharacter $\psi_{a]}$ is discontinuous, and a more detailed examination is needed.

It is convenient first to treat the case of non-negative, non-zero linear functionals $L$ and $M$. It is obvious that if $L$ and $M$ are non-negative, then $L * M$ is non-negative. The set $[\alpha, \alpha]$ being compact, we have $\lambda * \mu([\alpha, a])=\inf L * M(f)$, the infimum being taken over all $f \in \mathbb{C}(G)$ such that $f \geqq \psi_{a]}$ (see 1.6.2). Since the measure $\lambda$ is regular, we have

$$
\lambda([\alpha, a])=\inf \{\lambda(T): T \text { is open, } T \supset[\alpha, a]\} .
$$

Every such set $T$ contains an interval $[\alpha, u[$, where $u>a$, and hence

$$
\lambda([\alpha, a])=\inf \{\lambda([\alpha, u[): a<u \leqq \omega\} .
$$

Similarly, we see that

$$
\mu([\alpha, a])=\inf \{\mu([\alpha, u[): a<u \leqq \omega\} .
$$

Now let $\varepsilon$ be any positive real number. Since $\lambda$ and $\mu$ are additive measures, the preceding two sentences show that there exists an element $u \in] a, \omega]$ for which the following inequalities hold:

$$
\begin{aligned}
& \lambda(] a, u[)<\min \left(\frac{\varepsilon}{3 M(1)}, \frac{\varepsilon}{3}\right), \\
& \mu(] a, u[)<\min \left(\frac{\varepsilon}{3 L(1)} ; 1\right) .
\end{aligned}
$$

Since $G$ is normal, there exists $f \in \mathfrak{S}(G)$ such that $f(x)=1$ for $x \in[\alpha, \alpha], f(x)=0$ for $x \in[u, \omega]$, and $0 \leqq f(x) \leqq 1$ for $x \in G$. (See [9], p. 141, Theorem 5.9.) We now consider the function $f(\max (x, y))$ on $G \times G$. The following facts are easily verified:

$$
f(\max (x, y))= \begin{cases}1 & \text { for }(x, y) \in[\alpha, a] \times[\alpha, a], \\ 0 & \text { for }(x, y) \in G \times[u, \omega] \cup[u, \omega] \times G, \\ f(x) \text { for }(x, y) \in] \alpha, u[\times[\alpha, a], \\ f(y) \text { for }(x, y) \in[\alpha, a] \times] \alpha, u[.\end{cases}
$$

We now have, applying $3.2 .5,3.2 .3$, and 3.2 .4 :

$$
\begin{aligned}
\lambda * \mu([\alpha, a]) \leqq & L * M(f)=\int_{G} \int_{G} f(\max (x, y)) d \mu(y) d \lambda(x) \\
= & \lambda([\alpha, a]) \cdot \mu([\alpha, a])+\int_{] a, u[} f(x) d \lambda(x) \cdot \mu([\alpha, a]) \\
& +\int_{]_{a, u[}} f(y) d \mu(y) \cdot \lambda([\alpha, a])+\int_{]_{a, u}} \int_{]_{a, u}[} f(\max (x, y)) d \mu(y) d \lambda(x)
\end{aligned}
$$




$$
\begin{aligned}
& \leqq \lambda([\alpha, a]) \cdot \mu([\alpha, a])+\lambda(] a, u[) \cdot \mu([\alpha, a])+\mu(] a, u[) \cdot \lambda([\alpha, a]) \\
& +\lambda(] a, u[) \cdot \mu(] a, u[) \\
& <\lambda([\alpha, a]) \cdot \mu([\alpha, a])+\frac{\varepsilon}{3}+\frac{\varepsilon}{3}+\frac{\varepsilon}{3}=\lambda([\alpha, a]) \cdot \mu([\alpha, a])+\varepsilon .
\end{aligned}
$$

Since $\varepsilon$ is arbitrary, we infer that $\lambda * \mu([\alpha, a]) \leqq \lambda([\alpha, \alpha]) \cdot \mu([\alpha, \alpha])$.

To establish the reversed inequality, let $\varepsilon$ again be an arbitrary positive real number, and let $f \in \mathbb{S}(G)$ have the properties that $1 \geqq$ $f(x) \geqq \phi_{a]}(x)$ for $x \in G$ and $L * M(f)<\lambda * \mu([\alpha, a])+\varepsilon$. The existence of such a function $f$ follows at once from 1.6.2 and the non-negativity of $L * M$. It is obvious that $f(x) f(y) \leqq f(\max (x, y))$ for $(x, y) \in G \times G$. We now have

$$
\begin{aligned}
& \lambda([\alpha, a]) \cdot \mu([\alpha, a]) \leqq \int_{G} f(x) d \lambda(x) \cdot \int_{G} f(y) d \mu(y) \\
= & \int_{G} \int_{G} f(x) f(y) d \mu(y) d \lambda(x) \leqq \int_{G} \int_{G} f(\max (x, y)) d \mu(y) d \lambda(x) \\
= & L * M(f)<\lambda * \mu([\alpha, a])+\varepsilon .
\end{aligned}
$$

Since $\varepsilon$ is arbitrary, we have proved that

$$
\lambda * \mu([\alpha, \alpha])=\lambda([\alpha, a]) \cdot \mu([\alpha, a]) .
$$

We now prove that the mappings 3.2.2 are multiplicative for nonnegative $L$ and $M$. Since $\lambda, \mu$, and $\lambda * \mu$ are regular measures, there exists, for every positive integer $n$, a compact subset $C_{n}$ of $[\alpha, a[$ such that

$$
\lambda\left(\left[\alpha, a[)-\frac{1}{n}<\lambda\left(C_{n}\right), \quad \mu\left(\left[\alpha, a[)-\frac{1}{n}<\mu\left(C_{n}\right),\right.\right.\right.\right.
$$

and

$$
\lambda * \mu\left(\left[\alpha, a[)-\frac{1}{n}<\lambda * \mu\left(C_{n}\right)\right.\right.
$$

We may evidently suppose that $C_{n}=\left[\alpha, b_{n}\right]$ for some $b_{n}<a$. (If $a$ has an immediate predecessor $a_{-}$, so that $\left[\alpha, a\left[=\left[\alpha, a_{-}\right]\right.\right.$, we may refer to 3.2.6.) Then we have, applying 3.2.6:

$$
\begin{aligned}
\lambda * \mu([\alpha, a[) & =\lim _{n \rightarrow \infty} \lambda * \mu\left(C_{n}\right)=\lim _{n \rightarrow \infty}\left(\lambda\left(C_{n}\right) \cdot \mu\left(C_{n}\right)\right) \\
& =\left(\lim _{n \rightarrow \infty} \lambda\left(C_{n}\right)\right) \cdot\left(\lim _{n \rightarrow \infty} \mu\left(C_{n}\right)\right)=\lambda([\alpha, a[) \cdot \mu([\alpha, a[) .
\end{aligned}
$$

To establish the present theorem for arbitrary $L, M \in \widetilde{\mathfrak{S}}(G)$, we cite 1.6.5: $L=\left(L_{1}-L_{2}\right)+i\left(L_{3}-L_{4}\right), \quad M=\left(M_{1}-M_{2}\right)+i\left(M_{3}-M_{4}\right)$, where $L_{j}$ and $M_{k}$ are non-negative $(j, k=1, \cdots, 4)$. The relations 


$$
\begin{aligned}
& \lambda * \mu([\alpha, a])=\lambda([\alpha, a]) \cdot \mu([\alpha, a]) \quad(a \in G), \\
& \lambda * \mu([\alpha, a[)=\lambda([\alpha, a[) \cdot \mu([\alpha, a[) \quad(a \in[\alpha, \omega]),
\end{aligned}
$$

now follow from 3.2.6, 3.2.7, and the identity $(r \lambda+s \mu)(A)=r \lambda(A)+s \mu(A)$, valid for all $r, s \in K ; L, M \in \widetilde{\mathfrak{C}}(G)$, and Borel sets $A \subset G$. This completes the present proof.

3.3. TheOREM. Let $\pi$ be a homomorphism of $\tilde{\mathfrak{S}}(G)$ onto $K$. Then either there exists $b \in G$ such that $\pi(L)=\lambda([\alpha, b])$ for all $L \in \widetilde{\mathfrak{C}}(G)$, or there exists $b \in] \alpha, \omega]$ such that $\pi(L)=\lambda([\alpha, b[)$ for all $L \in \widetilde{\mathfrak{S}}(G)$.

Proof. It follows from 2.10 that $\pi\left(\varepsilon_{\alpha}\right)=1$. Let $x, y$ be elements of $G$. Then, using 2.12 , we have

$$
\pi\left(\varepsilon_{x}\right) \cdot \pi\left(\varepsilon_{y}\right)=\pi\left(\varepsilon_{x} * \varepsilon_{y}\right)=\pi\left(\varepsilon_{\max (x, y)}\right) .
$$

The function $p$ on $G$ such that $p(x)=\pi\left(\varepsilon_{x}\right)$ for all $x \in G$ is therefore a semicharacter of $G$. Theorem 1.8 asserts that either there exists $b \in G$ such that

$$
\pi\left(\varepsilon_{x}\right)=\psi_{b]}(x) \quad \text { for } \quad x \in G,
$$

or there exists $b \in] \alpha, \omega]$ such that

$$
\pi\left(\varepsilon_{x}\right)=\psi_{b[}(x) \quad \text { for } \quad x \in G .
$$

Suppose first that 3.3.1 holds. Applying $\pi$ to the left side of 2.9.1, we have

$$
\pi\left(\varepsilon_{a} * \lambda\right)=\pi\left(\varepsilon_{a}\right) \cdot \pi(\lambda)=\psi_{b]}(a) \pi(\lambda) .
$$

Applying $\pi$ to the right side of 2.9.1, we have

$$
\left.\pi\left(\lambda([\alpha, a]) \varepsilon_{a}\right)+\lambda^{] a, \omega]}\right)=\lambda([\alpha, a]) \psi_{b]}(a)+\pi\left(\lambda^{[a, \omega]}\right) .
$$

By 2.9.1, the last members of 3.3 .3 and 3.3 .4 are equal. We set $a=b$ in these expressions and equate them:

$$
\pi(\lambda)=\lambda([\alpha, b])+\pi\left(\lambda^{] b, \omega]}\right) .
$$

We next show that $\pi\left(\lambda^{\left.]^{b}, \omega\right]}\right)=0$. Here there are two cases. Suppose first that $b$ has no immediate successor and that $c$ is any element such that $c>b$. Then there is a $d$ such that $b<d<c$. It follows at once from 2.9.1 that $\varepsilon_{d} * \lambda^{[c, \omega]}=\lambda^{[c, \omega]}$. Since $\pi\left(\varepsilon_{d}\right)=0$, we have

$$
\pi\left(\lambda^{[c, \omega]}\right)=0 \text {. }
$$

To infer from this that $\pi\left(\lambda^{[b, \omega]}\right)=0$, we must use the continuity of $\pi$ in 
the norm of $\widetilde{\mathfrak{C}}(G)$. In fact,

$$
|\pi(L)| \leqq\|L\| \text { for all } L \in \widetilde{\mathfrak{C}}(G)
$$

([10], p. 69, Theorem). Let $\bar{\lambda}$ denote the total variation of $\lambda$ ([5], p. $459,1.2)$. It is easy to see that $\bar{\lambda}$ is regular. Thus, for every positive real number $\varepsilon$, there exists a compact subset of $] b, \omega]$ (which we may clearly take to be of the form $[c, \omega]$ with $c>b)$ such that $\bar{\lambda}(] b, c[)<\varepsilon$. Then we have

$$
\left\|\lambda^{[c, \omega]}-\lambda^{] b, \omega]}\right\|=\sup \left\{\left|\int_{]^{b, c}} f(x) d \lambda(x)\right|:\|f\| \leqq 1\right\} \leqq \bar{\lambda}(] b, c[)<\varepsilon .
$$

We infer from 3.3.6, 3.3.7, and 3.3.8 that $\left|\pi\left(\lambda^{j b, \omega]}\right)\right|<\varepsilon$, and hence $\pi\left(\lambda^{3], \omega]}\right)=0$.

Suppose next that $b$ has an immediate successor $b_{+}$. Then $\left.] b, \omega\right]=$ $\left[b_{+}, \omega\right]$. From 2.9.2, we have

$$
\varepsilon_{b_{+}} * \lambda^{\left[b_{+}, \omega\right]}=\lambda^{]^{b}+\omega\right]},
$$

and since $\pi\left(\varepsilon_{b_{+}}\right)=\psi_{b]}\left(b_{+}\right)=0$, we have

$$
\left.\pi\left(\lambda^{[b}{ }^{b}, \omega\right]\right)=0=\pi\left(\lambda^{b, \omega]}\right) .
$$

Therefore $\pi\left(\lambda^{[b, \omega]}\right)=0$ in both cases, and, returning to 3.3.5, we find

$$
\pi(\lambda)=\lambda([\alpha, b]) \quad \text { for all } \lambda \in \widetilde{\mathfrak{S}}(G) .
$$

This proves the present theorem in case 3.3.1 holds.

We have still to deal with the case in which 3.3.2 holds. If $b$ has an immediate predecessor, we are actually in the case 3.3.1. We therefore may suppose that $b$ has no immediate predecessor. Applying $\pi$ to both sides of 2.9.1, we have as before

$$
\pi(\lambda)=\lambda([\alpha, a])+\pi\left(\lambda^{] a, \omega]}\right) \quad \text { for } \quad a \in[\alpha, b[.
$$

Relations 2.9.2 and 3.3.2 imply that $\pi\left(\lambda^{[b, \omega]}\right)=0$. Hence

$$
\pi\left(\lambda^{] a, \omega]}\right)=\pi\left(\lambda^{] a, b}[)\right.
$$

for all $a \in\left[\alpha, b\left[\right.\right.$. An argument based on $\| \lambda^{3, b}[\|$, very like that used above, shows that for every positive real number $\varepsilon$, there exists $a_{0}<b$ such that $\left|\pi\left(\lambda^{] a, \omega]}\right)\right|<\varepsilon$ if $a_{0} \leqq a<b$. Since $\lambda$ is regular, there exists $a_{1}<b$ such that

$$
\mid \lambda([\alpha, \alpha])-\lambda\left(\left[\alpha, b[) \mid<\varepsilon \text { if } a_{1} \leqq a<b .\right.\right.
$$

From these facts and 3.3.10, we obtain the present theorem in case 3.3.2 holds. 
3.4. REMARK. It is interesting to compare 3.3 with the corresponding assertion for compact Abelian groups. Let $H$ be a compact Abelian group with group operation $x y$. Then $\tilde{\widetilde{c}}(H)$ is a convolution algebra, where

$$
\lambda * \mu(f)=\int_{H} \int_{H} f(x y) d \mu(y) d \lambda(x) \quad \text { for } \quad f \in \mathfrak{s}(H)
$$

If $H$ is infinite, then the homomorphisms of $\tilde{\widetilde{S}}(H)$ onto $K$ are enormously complicated, and in fact need not be described by characters of $H$ (see [12] for a detailed discussion).

\section{4. $\widetilde{\mathfrak{S}}(G)$ is semisimple.}

We establish first a preliminary result, which will also be of use in $\S 6$.

4.1. TheOREM. Let $f$ be an element of $\mathfrak{\subseteq}(G)$ and let $\varepsilon$ be a positive real number. Then there exists a finite subset $\left\{a_{j}\right\}_{j=0}^{m}$ of $G$, where

$$
\alpha=a_{0}<a_{1}<\cdots<a_{j}<a_{j+1}<\cdots<a_{m}=\omega,
$$

such that the oscillation of $f$ is less than $\varepsilon$ on each of the sets $\left.] a_{j-1}, a_{j}\right]$ $(j=1,2, \cdots, m)$.

Proof. The function $f$ is continuous. Hence, for all $x \in G$, there exists an interval neighborhood $U(x)$ such that $\left|f(y)-f\left(y^{\prime}\right)\right|<\frac{\varepsilon}{2}$ for all $y, y^{\prime} \in U(x)$. Since $G$ is compact, a finite number of these neighborhoods cover $G$. Let $U_{1}, U_{2}, \cdots, U_{p}$ be such a collection of neighborhoods.

Each $U_{j}$ has one of the following forms: $] u, v[;[u, v[;] u, v]$; $\{w\}(u<v)$. Whenever an interval $U_{j}$ can be written in one of the last three forms, let the elements $u, v$ or the element $w$ be considered as the endpoints of $U_{j}$. Otherwise, call $u, v$ the endpoints of $U_{j}$. There are at most $2 p$ distinct endpoints of the sets $U_{j}$ : we write them in increasing order as $a_{0}, a_{1}, \cdots, a_{m}$. Since $\alpha$ is in some $U_{j}$ and since the only types of open intervals containing $\alpha$ are $[\alpha, v[(\alpha<v)$ or $\{\alpha\}$ (if $\alpha$ is isolated), we must have $a_{0}=\alpha$. By the same token, we have $a_{m}=\omega$.

Now consider an arbitrary interval $\left.] a_{k-1}, a_{k}\right](k=1,2, \cdots, m)$. The point $a_{k}$ lies in some interval $U_{s}(s=1,2, \cdots, p)$. If $U_{s}$ is of the form ]$u_{s}, v_{s}[$ or $\left.] u_{s}, v_{s}\right]$, it is obvious that $a_{k-1} \geqq u_{s}$ and hence $\left.] a_{k-1}, a_{k}\right] \subset U_{s}$. If $U_{s}$ has the form $\left[u_{s}, v_{s}\left[\right.\right.$ with $u_{s}<a_{k}$, then it is again obvious that $\left.] a_{k-1}, a_{k}\right] \subset U_{s}$. In these cases, the oscillation of $f$ on $] a_{k-1}, a_{k}$ ] does not exceed $\varepsilon / 2$. If $U_{s}$ has the form $\left[a_{k}, v_{s}\right.$ [ or $\left\{a_{k}\right\}$, then since $U_{s}$ 
is open, $a_{k}$ has an immediate predessor, say $w$. If $a_{k-1}=w$, then ]$\left.a_{k-1}, a_{k}\right]=\left\{a_{k}\right\}$. Consider an interval $U_{t}$ that contains $w$. If $U_{t}$ has the form $\left[w, v_{t}[\right.$ or $\left.] u_{t}, w\right]$ or $\{w\}$, then $w=a_{k-1}$. In these three cases, the oscillation of $f$ on $] a_{k-1}, a_{k}$ ] is 0 . If $U_{t}$ has the form $] u_{t}, v_{t}[$ and does not have any of the three preceding forms, then we have $a_{k}<v_{t}$, $u_{t}<w$, and necessarily $u_{t} \leqq a_{k-1}$. Again it follows that $\left.] a_{k-1}, a_{k}\right] \subset U_{t}$, and the oscillation of $f$ on $] a_{k-1}, a_{k}$ ] does not exceed $\varepsilon / 2$. Since $\varepsilon / 2$ is less than $\varepsilon$, the lemma is proved.

Our next theorem shows that $\tilde{\widetilde{C}}(G)$ is semisimple.

4.2. Theorem. Let $L$ be an element of $\widetilde{\mathfrak{S}}(G)$ such that $\lambda([\alpha, a])=0$ for all $a \in G$. Then $L=0$.

Proof. Let $f$ be any function in $\widetilde{C}(G)$, let $\varepsilon$ be a positive real number, and let $\left\{a_{j}\right\}_{j=0}^{m}$ be as in 4.1. Let $p$ be the function on $G$ such that

$$
p(x)=\left\{\begin{array}{l}
f(\alpha) \text { for } x=\alpha, \\
f\left(a_{k}\right) \text { for } a_{k-1}<x \leqq a_{k} \quad(k=1,2, \cdots, m) .
\end{array}\right.
$$

Then $p$ is Borel measurable and bounded and hence is in $\mathbb{2}_{1}(\lambda)$. Our hypothesis on $\lambda$ implies that $\lambda(\{a\})=0$ and that $\left.\left.\lambda(] a_{k-1}, a_{n}\right]\right)=0 \quad(k=1,2$, $\cdots, m)$. Consequently, $\int_{G} p(x) d \lambda(x)=0$. On the other hand, we have $|p(x)-f(x)|<\varepsilon$ for all $x \in G$. Therefore

$$
\begin{aligned}
|L(f)| & =\left|\int_{G} f(x) d \lambda(x)\right|=\left|\int_{G} f(x) d \lambda(x)-\int_{G} p(x) d \lambda(x)\right| \\
& \leqq \int_{G}|f(x)-p(x)| d \bar{\lambda}(x) \leqq \varepsilon \bar{\lambda}(G)
\end{aligned}
$$

Since $\varepsilon$ is an arbitrary positive real number, it follows that $L(f)=0$, and therefore $L=0$.

4.3. THeOREM. Let $L$ be an element of $\tilde{\widetilde{S}}(G)$ such that $\lambda(\{\omega\})=0$ and $\lambda([\alpha, a[)=0$ for all $a \in] \alpha, \omega]$. Then $L=0$.

The proof of this theorem differs only trivially from that of 4.2.

4.4. THEOREM. The Banach algebra $\widetilde{\mathfrak{S}}(G)$ is simisimple. If $L \in \widetilde{\mathfrak{C}}(G)$ and $L \neq 0$, then the image of $L$ under some homomorphism 3.2 .1 is different from zero. If the image of $L$ under every homomorphism 3.2.2 is zero, then $L=t \varepsilon_{\omega}$ for some $t \in K$.

Proof. The second statement of this theorem merely repeats 4.2. The first statement follows from the second. To prove the third state- 
ment, let $t=\lambda(\{\omega\})$. Then $\left(\lambda-t \varepsilon_{\omega}\right)([\alpha, a[)=0$ for all $a \in] \alpha, \omega]$, and $\left(\lambda-t \varepsilon_{\omega}\right)(\{\omega\})=0$. We now appeal to 4.3.

\section{5. $\hat{G}$ as the maximal ideal space of $\tilde{\mathfrak{S}}(G)$.}

5.1. Theorems 3.2 and 3.3 identify completely the homomorphisms of $\tilde{\widetilde{S}}(G)$ onto $K$. In order to study the space of all these homomorphisms, we introduce some new notation. For all $a \in G$, let $\boldsymbol{a}$ denote the homomorphism 3.2.1: $\boldsymbol{a}(L)=\lambda([\alpha, a])$. Let $\boldsymbol{G}$ denote the set of all homomorphisms $\boldsymbol{a}$. For $\lambda \in \widetilde{\mathbb{S}}(G)$, we define the function $\hat{\lambda}$ on $\boldsymbol{G}$ as usual : $\hat{\lambda}(\boldsymbol{a})=\boldsymbol{a}(L)$ for all $\boldsymbol{a} \in \boldsymbol{G}$. For $a \in] \alpha, \omega]$, let $\boldsymbol{a}^{\prime}$ denote the homomorphism 3.2.2: $\boldsymbol{a}^{\prime}(L)=\lambda\left[\alpha, \alpha[)\right.$. Let $\boldsymbol{G}^{\prime}$ denote the set of all homomorphisms $\boldsymbol{a}^{\prime}$. For $\lambda \in \tilde{\mathfrak{S}}(G)$, we define the function $\hat{\lambda}$ on $\boldsymbol{G}^{\prime}$ as usual : $\hat{\lambda}\left(\boldsymbol{a}^{\prime}\right)=\boldsymbol{a}^{\prime}(L)$ for all $\boldsymbol{a}^{\prime} \in \boldsymbol{G}^{\prime}$. By an abuse of notation, we identify $\boldsymbol{G} \cup \boldsymbol{G}^{\prime}$ with the semigroup $\hat{G}$ of all semicharacters of $G$ (1.8 and 1.12). Theorems 3.2 and 1.8 of course suggest this step. The function $\hat{\lambda}$ on $\hat{G}=\boldsymbol{G} \cup \boldsymbol{G}^{\prime}$ is called the Fourier transform of $L$.

5.2. Before going further, we must agree on certain identifications that may have to be made between $\boldsymbol{G}$ and $\boldsymbol{G}^{\prime}$. If $a \in G$ and $a$ has an immediate successor $a_{+}$, then $[\alpha, \alpha]=\left[\alpha, a_{+}\left[\right.\right.$, and hence $\boldsymbol{a}=\boldsymbol{a}_{+}^{\prime}$. Equivalently, if $a \in] \alpha, \omega]$ and $a$ has an immediate predecessor $a_{-}$, then $[\alpha, a[=$ $\left[\alpha, a_{-}\right]$, and $\boldsymbol{a}^{\prime}=\boldsymbol{a}_{-}$. For all such $\left.\left.a \in\right] \alpha, \omega\right]$, we agree to identify the point $\boldsymbol{a}^{\prime}$ with the point $\boldsymbol{a}_{-}$.

5.3. For $u, v \in G$, we define $[\boldsymbol{u}, \boldsymbol{v}]$ as the set of all $\boldsymbol{c} \in \boldsymbol{G}$ such that $u \leqq c \leqq v$. The sets $\left[\boldsymbol{u}, \boldsymbol{v}\left[,\left[\boldsymbol{u}^{\prime}, \boldsymbol{v}^{\prime}\right]\right.\right.$, etc., are defined similarly.

5.4. The Gel'fand topology for $\hat{G}$ is the weakest topology (that is, the topology with the smallest family of open sets) that makes all of the functions $\hat{\lambda}$ continuous. It is well known that $\hat{G}$ is a compact Hausdorff space in this topology $([10]$, p. 52, Theorem 19B). We now describe the Gel'fand topology for $\hat{G}$.

5.5. TheOREM. The point $\omega$ is isolated in $\hat{G}$. If $b \in[\alpha, \omega[$ and $b$ has no immediate successor, then a complete family of neighborhoods of $\boldsymbol{b}$ consists of all sets of the form

$$
\left.\left.\left[\boldsymbol{b}, \boldsymbol{c}[\cup] \boldsymbol{b}^{\prime}, \boldsymbol{c}^{\prime}\right] \quad \text { where } c \in\right] b, \omega\right] .
$$

If $b \in\left[\alpha, \omega\left[\right.\right.$ and $b$ has an immediate successor $b_{+}$, then $\boldsymbol{b}=\boldsymbol{b}_{+}^{\prime}$ is isolated in $\hat{G}$. If $b \in] \alpha, \omega]$ and $b$ has no immediate predecessor, then a complete 
family of neighborhoods of $\boldsymbol{b}^{\prime}$ consists of all sets of the form

$$
] \boldsymbol{a}^{\prime}, \boldsymbol{b}^{\prime}\right] \cup[\boldsymbol{a}, \boldsymbol{b}[\quad \text { where } a \in[\alpha, b[\text {. }
$$

It $b$ has an immediate predecessor $b_{-}$, then $\boldsymbol{b}^{\prime}=\boldsymbol{b}_{-}$, and is isolated.

Proof. We use repeatedly the fact that all $\hat{\lambda}$ must be continuous on $G$. The function $\hat{\varepsilon}_{\omega}$ is 0 everywhere on $\hat{G}$ except at $\omega$, and $\hat{\varepsilon}_{\omega}(\omega)=1$. Hence $\omega$ is isolated.

Consider next any point $\boldsymbol{b}$ such that $\alpha \leqq b<\omega$. If $b$ has no immediate successor, there exists, for every open set $T$ containing $[\alpha, b]$, an element $c$ such that $c>b$ and $[\alpha, b] \subset[\alpha, c[\subset T$. Every measure $\lambda \in \mathbb{C}(\tilde{G})$ is regular, and hence we can find a $c_{0}>b$ such that

$$
\mid \lambda([\alpha, b])-\lambda([\alpha, c)[\mid<\varepsilon
$$

for all $c$ such that $b<c \leqq c_{0}$, $\varepsilon$ being an arbitrary positive real number. This means that

$$
\left|\hat{\lambda}(\boldsymbol{b})-\hat{\lambda}\left(\boldsymbol{c}^{\prime}\right)\right|<\varepsilon \quad \text { if } \quad b<c \leqq c_{0} .
$$

If $\lambda$ is non-negative, 5.5.3 clearly implies that $|\lambda([\alpha, b])-\lambda([\alpha, c])|<\varepsilon$ for all $c$ such that $b \leqq c \leqq c_{0}$. Since $\lambda$ is a linear combination of four nonnegative measures, we now have the following result.

5.5.5. Let $b \in[\alpha, \omega[$ and let $b$ have no immediate successor. Let $\lambda \in \widetilde{\mathfrak{C}}(G)$, and let $\varepsilon$ be any positive real number. Then there is a $c_{0}>b$ such that $|\hat{\lambda}(\boldsymbol{b})-\hat{\lambda}(\boldsymbol{c})|<\varepsilon$ if $b \leqq c<c_{0}$ and $\left|\hat{\lambda}(\boldsymbol{b})-\hat{\lambda}\left(\boldsymbol{c}^{\prime}\right)\right|<\varepsilon$ if $b<c \leqq c_{0}$.

If $b$ has an immediate successor, $b_{+}$, then we have

$$
\hat{\varepsilon}_{b}(\boldsymbol{x})-\hat{\varepsilon}_{b_{+}}(\boldsymbol{x})= \begin{cases}1 & \text { if } \boldsymbol{x}=\boldsymbol{b}=\boldsymbol{b}_{+}^{\prime}, \\ 0 & \text { elsewhere on } \hat{G} .\end{cases}
$$

Since the function $\hat{\varepsilon}_{b}-\hat{\varepsilon}_{b_{+}}$is continuous on $\hat{G}$, the point $\boldsymbol{b}=\boldsymbol{b}_{+}^{\prime}$ is isolated.

We next consider a point $\boldsymbol{b}^{\prime} \in \hat{G}$ such that $b$ has no immediate predecessor. Then $[\alpha, b[$ is a nonclosed open subset of $G$, and for every closed subset $F$ of $[\alpha, b[$, there exists $c<b$ such that $F \subset[\alpha, c] \subset[\alpha, b[$. If $\lambda \in \widetilde{\mathfrak{S}}(G)$, then $\lambda$ is regular, and we see just as in 5.5.5 that:

5.5.7. $\hat{\lambda}\left(\boldsymbol{c}^{\prime}\right)$ is arbitrarily close to $\hat{\lambda}\left(\boldsymbol{b}^{\prime}\right)$ if $c_{0}<c \leqq b$ and $\hat{\lambda}(\boldsymbol{c})$ is arbitrarily close to $\hat{\lambda}\left(\boldsymbol{b}^{\prime}\right)$ if $c_{0} \leqq c<b$ (here $c_{0}$ is an appropriately chosen element $<b$ ).

The case in which $b$ has an immediate predecessor has already been dealt with. 
The topology imposed on $\hat{G}$ by the neighborhood system 5.5.1 and 5.5.2 (and with isolated points as described) is obviously a Hausdorff topology. In 5.5.5, 5.5.6, and 5.5.7, we have shown that every function $\hat{\lambda}$ is continuous on $\hat{G}$ in this topology. From 5.4, we see that the Gel'fand topology is weaker than or equivalent to the topology just described.

To show that this topology is precisely the Gel'fand topology, consider any $b, c \in G$ such that $\alpha \leqq b<c \leqq \omega$ and such that $b$ has no immediate successor. It is easy to see that

$$
\hat{\varepsilon}_{b}(\boldsymbol{x})-\hat{\varepsilon}_{c}(\boldsymbol{x})= \begin{cases}1 & \text { if } \boldsymbol{x} \in\left[\boldsymbol{b}, \boldsymbol{c}[\cup] \boldsymbol{b}^{\prime}, \boldsymbol{c}^{\prime}\right], \\ 0 & \text { elsewhere on } G .\end{cases}
$$

Hence all of the neighborhoods of $\boldsymbol{b}$ enumerated in 5.5.1 are necessarily open in the Gel'fand topology. Since $c$ is the immediate successor of $b$ if and only if $b$ is the immediate predecessor of $c$, the same function $\hat{\varepsilon}_{b}-\hat{\varepsilon}_{c}$ shows that all of the neighborhoods of $\boldsymbol{b}^{\prime}$ enumerated in 5.5.2 must be open in the Gel'fand topology. Points with immediate successors and the point $\omega$ have already been dealt with: such points must be isolated in the Gel'fand topology for $\hat{G}$. This completes the present proof.

5.6. REMARK. Since $\tilde{\widetilde{C}}(G)$ has the unit $\varepsilon_{\alpha}(2.10), \hat{G}$ must be compact. Thus the topology of 5.5 is a compact Hausdorff topology. This fact could of course be established by a direct examination of $\hat{G}$.

5.7. The mapping $L \rightarrow \hat{\lambda}$ is a linear mapping of $\hat{夭}(G)$ into the function space $\mathfrak{S}(\hat{G})$ that changes convolution into pointwise multiplication. That is, $L * M \rightarrow(\lambda * \mu)^{\wedge}=\hat{\lambda} \cdot \hat{\mu}$ for all $L, M \in \widetilde{\mathcal{C}}(G)$, where $\hat{\lambda} \cdot \hat{\mu}$ is the pointwise product of $\hat{\lambda}$ and $\hat{\mu}$ on $\hat{G}$. This follows at once from 3.2. Theorem 4.4 shows that this mapping is an algebraic isomorphism. The result of the present section is to describe the (unique) compact Hausdorff topology on $\hat{G}$ under which the functions $\hat{\lambda}$ are continuous. Thus in studying algebraic properties of $\widetilde{\widetilde{S}}(G)$, we may consider the subspace of $\hat{\mathfrak{S}}(G)$ consisting of all $\hat{\lambda}$. In 6.7 and 6.9 , we will give a more precise description of these functions.

5.8. The Stone (or kernel-hull) topology for $\hat{G}([10]$, p. 56) is identical with the Gel'fand topology. A neighborhood of $\boldsymbol{x} \in \hat{G}$ in the Stone topology consists of all $\boldsymbol{y}$ such that $\hat{\lambda}(\boldsymbol{y}) \neq 0$, where $\hat{\lambda}(\boldsymbol{x}) \neq 0$. It is clear that the Stone topology is weaker than or equal to the Gel'fand topology, and since the functions $\hat{\varepsilon}_{b}-\hat{\varepsilon}_{c}$ are different from 0 exactly on 
the neighborhoods 5.5.1 and 5.5.2, the two topologies coincide.

\section{The Herglotz-Bochner theorem for $\tilde{\mathfrak{S}}(G)$.}

6.1. Weil's generalization to locally compact Abelian groups of the Herglotz-Bochner theorem (see [10], pp. 141-142, Theorem 36A) gives an intrinsic characterization (positive definiteness and continuity) of all functions on the dual group that are Fourier-Stieltjes transforms of finite non-negative regular Borel measures. We here give two analogues of the Herglotz-Bochner theorem for the algebra $\tilde{\mathfrak{S}}(G)$.

6.2. Let $\lambda$ be a non-negative measure in $\widetilde{\mathfrak{S}}(G)$. Then the function $\hat{\lambda}$ is continuous, real-valued, and non-negative on $\hat{G}$. It is also nondecreasing in the sense that $\hat{\lambda}(\boldsymbol{a}) \leqq \hat{\lambda}(\boldsymbol{b})$ and $\hat{\lambda}\left(\boldsymbol{a}^{\prime}\right) \leqq \hat{\lambda}\left(\boldsymbol{b}^{\prime}\right)$ if $a \leqq b$. We shall show that these properties completely characterize Fourier transforms of non-negative measures. In fact if $h$ is a continuous, real-valued, nonnegative function on $\hat{G}$ such that $h(\boldsymbol{a}) \leqq h(\boldsymbol{b})$ for $a \leqq b$, and $h(\boldsymbol{\omega})>0$, then $h=\hat{\lambda}$ for some non-zero $\lambda \in \hat{\mathfrak{S}}(G)$ such that $\lambda \geqq 0$. The proof requires a number of steps, which we state as separate theorems.

6.3. THEOREM. Let $h$ be a continuous function on $\hat{G}$ that is realvalued and non-decreasing on $\boldsymbol{G}$. Then $h$ is also real-valued and nondecreasing on $\boldsymbol{G}^{\prime}$.

Proof. It is first clear that $h$ is real-valued on $\boldsymbol{G}^{\prime}$, since $\boldsymbol{G}$ is dense in $\hat{G}$. Let $a, b$ be elements of $G$ such that $a<b$, and let $\varepsilon$ be a positive real number. There exists an element $c<a$ such that $\left|h\left(\boldsymbol{a}^{\prime}\right)-h(\boldsymbol{x})\right|<\varepsilon$ for all $\boldsymbol{x}$ such that $c \leqq x<a$ (see 5.5.2). This holds trivially if $a$ has an immediate predecessor. Similarly, there exists an $e<b$ such that $\left|h\left(\boldsymbol{b}^{\prime}\right)-h(\boldsymbol{y})\right|<\varepsilon$ for all $\boldsymbol{y}$ such that $e \leqq y<b$. If we choose $e \geqq a$, then, as $h$ is non-decreasing on $\boldsymbol{G}$, all of the numbers $h(\boldsymbol{x})$ are less than or equal to all of the numbers $h(\boldsymbol{y})$, and it follows that $h\left(\boldsymbol{a}^{\prime}\right) \leqq h\left(\boldsymbol{b}^{\prime}\right)$.

Given a function $h$ as in 6.2, we must recapture the measure $\lambda$, or, equivalently, the linear functional $L$, whose Fourier transform is $h$. For this purpose, we introduce a Riemann integral with respect to $h$.

6.4. Definition. Let $h$ be any real-valued, non-decreasing function on $\boldsymbol{G}$. Let $\Delta$ denote a finite subset $\left\{a_{0}, a_{1}, \cdots, a_{m}\right\}$ of $G$, such that $a_{0}=\alpha, \quad a_{m}=\omega$, and $a_{j-1}<a_{j}(j=1, \cdots, m)$. For an arbitrary complexvalued function $f$ on $G$, let

$$
S(f, \Delta)=\sum_{j=1}^{m} f\left(a_{j}\right)\left(h\left(\boldsymbol{a}_{j}\right)-h\left(\boldsymbol{a}_{j-1}\right)\right) .
$$


6.5. THeOREM. Let $f$ be a continuous function on $G$. Then there exists a unique number $L(f)$ such that for every $\varepsilon>0$ there exists a $A_{0}$ as in 6.4 with the property that $|L(f)-S(f, \Delta)|<\varepsilon$ for all $\Delta \supset \Delta_{0}$. We write this relation as $L(f)=\lim _{\Delta} S(f, \Delta)$.

Proof. Let $\beta=h(\omega)-h(\alpha)$. If $\beta=0$, then $S(f, \Delta)=0$ for all $\Delta$ and there is really nothing to prove. Otherwise, let $\theta$ be an arbitrary positive real number. Then, by 4.1 , there exists a $\Delta=\left\{a_{j}\right\}_{j=0}^{m}$ such that the oscillation of $f$ is less than $\beta^{-1} \theta$ in each of the sets $\left.] a_{j-1}, a_{j}\right] \quad(j=1,2$, $\cdots, m)$. Suppose now that $\Gamma$ is a finite subset of $G$ such that $\Gamma \supset \Delta$. We shall prove that

$$
|S(f, \Delta)-S(f, \Gamma)|<\theta
$$

Write $\Gamma=\left\{b_{k}\right\}_{k=0}^{r}, b_{k-1}<b_{k}$, and suppose that $b_{s}=a_{1}, b_{k}<a_{1}$ for $k<s$. Then we have

$$
\begin{aligned}
& \left|\sum_{k=1}^{s} f\left(b_{k}\right)\left(h\left(\boldsymbol{b}_{k}\right)-h\left(\boldsymbol{b}_{k-1}\right)\right)-f\left(a_{1}\right)\left(h\left(\boldsymbol{a}_{1}\right)-h\left(\boldsymbol{a}_{0}\right)\right)\right| \\
& \quad=\left|\sum_{k=1}^{s}\left(f\left(b_{k}\right)-f\left(a_{1}\right)\right)\left(h\left(\boldsymbol{b}_{k}\right)-h\left(\boldsymbol{b}_{k-1}\right)\right)\right| \\
& \quad \leqq \beta^{-1} \theta\left(\sum_{k=1}^{s}\left(h\left(\boldsymbol{b}_{k}\right)-h\left(\boldsymbol{b}_{k-1}\right)\right)\right)=\beta^{-1} \theta\left(h\left(\boldsymbol{a}_{1}\right)-h\left(\boldsymbol{a}_{0}\right)\right) .
\end{aligned}
$$

If $h\left(\boldsymbol{a}_{1}\right)-h\left(\boldsymbol{a}_{0}\right)$ is positive, it is clear that the inequality in 6.5.2 is strict. Estimates similar to 6.5 .2 obviously hold for the $b$ 's lying in the intervals $\left.\left.\left.] a_{1}, a_{2}\right], \cdots,\right] a_{m-1}, a_{m}\right]$. Adding these estimates together, we obtain the result that

$$
|S(f, \Delta)-S(f, \Gamma)|<\left[\sum_{j=1}^{m}\left(h\left(\boldsymbol{a}_{)}\right)-h\left(\boldsymbol{a}_{j-1}\right)\right)\right] \beta^{-1} \theta=\theta .
$$

the strict inequality holding because some $h\left(\boldsymbol{a}_{j}\right)-h\left(\boldsymbol{a}_{j-1}\right)$ is positive. This is just 6.5.1.

Let $\Delta_{n}$ be a subset of $G$ as in 6.4 such that $\left|S(f, \Delta)-S\left(f, \Delta_{n}\right)\right|<n^{-1}$ for all $\Delta \supset \Delta_{n}$, and let $\Gamma_{n}=\bigcup_{j=1}^{n} \Delta_{j}(n=1,2,3, \cdots)$. Then $\left\{S\left(f, \Gamma_{n}\right)\right\}_{n=1}^{\infty}$ is a Cauchy sequence of complex numbers and hence has a limit, which we take as $L(f)$. If $\varepsilon$ is a positive real number, then there exists an $n>3 / \varepsilon$ such that $\left|L(f)-S\left(f, \Gamma_{n}\right)\right|<\varepsilon / 3$. If $\Delta \supset \Gamma_{n}$, then $\Delta \supset \Delta_{n}$, so that $\left|S(f, \Delta)-S\left(f, \Gamma_{n}\right)\right|<2 / n$. Thus $|L(f)-S(f, \Delta)|<\varepsilon$, as was to be proved. The uniqueness of $L(f)$ is proved by a standard argument, which we omit.

6.6. Theorem. The function $L$ defined in 6.5 for all $f \in \mathbb{C}(G)$ is a non-negative linear functional on $\mathfrak{S}(G)$. 
Proof. Since $h$ is real and non-decreasing, it is clear that $S(\Delta, f)$ is real and non-negative for all real $f \in \mathfrak{S}(G)$ that are nonnegative and all $\Delta$ as in 6.4. Hence the limit $L(f)$ of these numbers is non-negative. The linearity of $L$ follows at once from 6.5 and the obvious equality $S(\Delta, u f+v g) \equiv u S(\Delta, f)+v S(\Delta, g)$, valid for all complex numbers $u$, $v$, all $f, g \in \mathbb{C}(G)$, and $\Delta$ as in 6.4 .

We can now state and prove our main theorem.

6.7. Theorem. Let $h$ be a continuous function on $\hat{G}$ that is realvalued, non-negative, and non-decreasing on $\boldsymbol{G}$. Let $L$ be the non-negative linear functional associated with $h$ as in 6.5. Let $\lambda$ be the measure associated with $L$ as in 1.6.1. Then $h$ is the Fourier transform of $\lambda+h(\boldsymbol{\alpha}) \varepsilon_{\alpha}:$

$$
h=\hat{\lambda}+h(\boldsymbol{\alpha}) \hat{\varepsilon}_{\alpha}=\hat{\lambda}+h(\boldsymbol{\alpha}) .
$$

Proof. Since $\hat{\lambda}$ and $h$ are completely determined by their behavior on the dense subset $\boldsymbol{G}$ of $\hat{G}$, we have only to show that 6.7.1 holds on G. That is, we must show that

$$
\hat{\lambda}(\boldsymbol{a})=\lambda([\alpha, a])=h(\boldsymbol{a})-h(\boldsymbol{\alpha}) \text { for all } \quad a \in G .
$$

If $a \in G$ and $a$ has an immediate successor, then the function $\psi_{a]}$ is continuous, and by the definition of $\lambda$ given in 1.6.2, we have $\lambda([\alpha, a])=$ $L\left(\psi_{a]}\right)$. If $\Delta$ is any finite subset of $G$ as in 6.4 that contains $a$, then it is plain that $S\left(\psi_{a]}, \Delta\right)=h(\boldsymbol{a})-h(\boldsymbol{\alpha})$. This implies that $L\left(\psi_{a]}\right)=h(\boldsymbol{a})-h(\boldsymbol{\alpha})$, that is, that 6.7.2 holds for this value of $a$.

If $a$ has no immediate successor, then, for every positive real number $\varepsilon$ and every $b>a, b \in G$, there exists a non-negative real-valued function $f \in \mathfrak{C}(G)$ such that $f(x)=1$ for $x \leqq a, f(x)=0$ for $x \geqq b, 0 \leqq f(x) \leqq 1$ for $x \in G$, and

$$
|\lambda([\alpha, a])-L(f)|<\frac{\varepsilon}{3}
$$

This follows at once from 1.6.2 and the fact that $G$ is a normal topological space. Now let $\Delta$ be any finite subset of $G$ as in 6.4 that contains $a$ and $b$. The inequalities

$$
h(\boldsymbol{a})-h(\boldsymbol{\alpha}) \leqq S(f, \Delta) \leqq h(\boldsymbol{b})-h(\boldsymbol{\alpha})
$$

obviously hold. Since $h$ is continuous on $\hat{G}$, we can choose the element $b>a$ such that $0 \leqq h(\boldsymbol{b})-h(\boldsymbol{a})<\varepsilon / 3$. By 6.5 , there exists a finite subset $\Gamma$ of $G$ such that $\Gamma \supset \Delta$ and

$$
|S(f, \Gamma)-L(f)|<\frac{\varepsilon}{3} .
$$


Combining 6.7.3, 6.7.4, and 6.7.5, we have

$$
|\lambda([\alpha, \alpha])-(h(\boldsymbol{a})-h(\boldsymbol{\alpha}))|<\varepsilon .
$$

Since $\varepsilon$ is arbitrary, we have proved 6.7.2.

6.8. REMARK. Theorem 6.7 is an analogue of the Herglotz-Bochner theorem, since it characterizes in a simple way those functions on $\hat{G}$ that are Fourier transforms of non-negative measures in $\tilde{\mathfrak{s}}(G)$. We can also obtain an exact analogue of the Herglotz-Bochner theorem in terms of positive definite functions. A function $p$ on $\hat{G}$ is said to be positive definite if

$$
\sum_{j=1}^{m} \sum_{k=1}^{m} \xi_{j} \bar{\xi}_{k} p\left(\chi_{j} \chi_{k}\right) \geqq 0
$$

for all complex numbers $\xi_{1}, \cdots, \xi_{m}$ and all distinct $\chi_{1}, \cdots, \chi_{m}$ in $\hat{G}$. If $\lambda$ is a non-negative measure in $\widetilde{\mathfrak{S}}(G)$, then we have

$$
\begin{aligned}
\sum_{j=1}^{m} \sum_{k=1}^{m} \xi_{j} \bar{\xi}_{k} \hat{\lambda}\left(\chi_{j} \chi_{k}\right) & =\sum_{j=1}^{m} \sum_{k=1}^{m} \xi_{j} \bar{\xi}_{k} \int_{G} \chi_{j}(x) \chi_{k}(x) d \lambda(x) \\
& =\int_{G}\left|\sum_{j=1}^{m} \xi_{j} \chi_{j}(x)\right|^{2} d \lambda(x) \geqq 0 .
\end{aligned}
$$

Hence $\hat{\lambda}$ is continuous and positive definite in the sense of 6.8.1. Conversely, let $p$ be a continuous function on $\hat{G}$ that satisfies 6.8.1. Let $a, b \in G$ and let $a<b$. For $m=2, \chi_{1}=\psi_{a]}, \chi_{2}=\psi_{b]}, \xi_{1}=1$, and $\xi_{2}=-1$, the the inequality 6.8 .1 obviously reduces to

$$
-p\left(\psi_{a]}\right)+p\left(\psi_{b]}\right) \geqq 0 .
$$

Writing $p\left(\psi_{x]}\right)=p(\boldsymbol{x})$ for $x \in G$, we have $p(\boldsymbol{a}) \leqq p(\boldsymbol{b})$. From 6.8.1, we also see that $p$ is non-negative. That is, $p$ is continuous and non-decreasing on $\boldsymbol{G}$ and hence is the Fourier transform of a non-negative measure (6.7). Monotonicity is a much easier property to verify, in applications, than the inequality 6.8 .1 , so that the present characterization of Fourier transforms of nonnegative measures as continuous, positive definite functions is perhaps only a curiosity.

6.9. REMARK. Theorem 6.7 permits us to characterize general Fourier transforms $\hat{\lambda}$, where $\lambda$ is an arbitrary complex-valued measure in $\widetilde{\widetilde{C}}(G)$, as being continuous functions on $G$ that are linear combinations of continuous, real-valued, non-decreasing functions. However, there is another characterization of the functions $\hat{\lambda}$, more intrinsic in nature. Namely, let $p$ be a function on $\hat{G}$ and let $a, b$ be elements of $G$ such 
that $a \leqq b$. We define the variation of $p$ on the interval $[\boldsymbol{a}, \boldsymbol{b}]$ as the supremum of all numbers

$$
\sum_{j=1}^{m}\left|p\left(\boldsymbol{\alpha}_{j}\right)-p\left(\boldsymbol{\alpha}_{j-1}\right)\right|
$$

taken over all finite sets $a=a_{0}<a_{1}<\cdots<a_{m}=b$ (if $a=b$, we take the variation as 0$)$. We write this variation as $V(p: \boldsymbol{a}, \boldsymbol{b})$. One can then prove that a function $q$ on $\hat{G}$ is the Fourier transform of some measure in $\widetilde{\mathfrak{C}}(G)$ if and only if $q$ is continuous and $V(q: \boldsymbol{\alpha}, \boldsymbol{\omega})$ is finite. The proof is suggested by standard arguments from the elementary theory of functions of a real variable (see for example [11], pp. 215-223). In the non-trivial direction, the proof is carried out by showing that every continuous real-valued function of finite variation on $\hat{G}$ is the difference of two continuous, real-valued, non-decreasing functions on $\hat{G}$. We omit the details.

\section{An application to the theory of probability.}

7.1. Theorem 6.7 has applications to the theory of probability. Let $\Phi$ be a random variable defined on a probability space $(Y, \pi)$ with values in $G$. The function $d$ on $\hat{G}$, defined by

$$
\left\{\begin{array}{cl}
d(\boldsymbol{a})=\pi\{y: y \in Y, \Phi(y) \leqq a\} & \text { for } \quad a \in G, \\
d\left(\boldsymbol{a}^{\prime}\right)=\pi\{y: y \in Y, \Phi(y)<a\} & \text { for } \quad a \in] \alpha, \omega],
\end{array}\right.
$$

is obviously non-decreasing on $\boldsymbol{G}$ and $\boldsymbol{G}^{\prime}$. Under some obvious hypotheses on $\pi$ and $\Phi$, this function $d$ is continuous on $\hat{G}$ and hence is the Fourier transform of a probability measure $\lambda$ in $\hat{\hat{S}}(G)(6.7)$. It is clear that $\lambda$ is non-negative, and since $d(\omega)=1$, we must have $\lambda(G)=1$, that is, $\lambda$ is a probability measure on the Borel sets of $G$. If $\Phi_{j}$ are independent random variables as above with corresponding probability measures $\lambda_{j} \in \widetilde{\mathcal{S}}(G)$ $(j=1, \cdots, m)$, then the probability corresponding to the product $\Phi_{1} \cdots \Phi_{m}$ is the convolution $\lambda_{1} * \cdots * \lambda_{m}$. Thus the arithmetic of independent sets of random variables is just the arithmetic of the set of all continuous, nonnegative, non-decreasing functions $p$ on $\hat{G}$ such that $p(\omega)=1$. The operation is of course pointwise multiplication on $\hat{G}$. If we denote the set of all probability measures in $\tilde{\mathfrak{S}}(G)$ by $\mathfrak{s}$, then the set of function on $\hat{G}$ that we are now considering is exactly $\hat{\mathfrak{F}}$. In the case of a finite semigroup $G$, the arithmetic arising in this way has been studied in detail in another place [6].

We proceed to a description of some of the properties of $\mathfrak{P}$ and $\hat{\mathfrak{F}}$. 
7.2. It is clear that $\mathfrak{P}$ has a unit, namely $\varepsilon_{\alpha}(2.10)$. Since the measure $\varepsilon_{\omega}$ has the property that

$$
\hat{\varepsilon}_{\omega}(\boldsymbol{x})=\left\{\begin{array}{lll}
0 & \text { if } \quad \boldsymbol{x} \neq \boldsymbol{\omega}, \\
1 & \text { if } \quad \boldsymbol{x}=\boldsymbol{\omega},
\end{array}\right.
$$

it follows that $\varepsilon_{\omega} * \lambda=\varepsilon_{\omega}$ for all $\lambda$ such that $\lambda(G)=1$. Hence $\varepsilon_{\omega}$ is a zero in the set $\mathfrak{s}$.

7.3. We next identify the idempotent elements of $\mathfrak{F}$. If $\lambda * \lambda=\lambda$, where $\lambda \in \widetilde{S}(G)$, then $\hat{\lambda}^{2}=\hat{\lambda}$, and $\hat{\lambda}$ assumes only the values 0 and 1 . If $\lambda \in \mathfrak{P}$, then $\hat{\lambda}$ is nondecreasing on $\boldsymbol{G}$ and $\lambda(\boldsymbol{\omega})=1$. The requirement of continuity makes it obvious that there exists an element $b \in G$ such that $\hat{\lambda}(\boldsymbol{a})=0$ for $a<b, \hat{\lambda}(\boldsymbol{a})=1$ for $a \geqq b, \hat{\lambda}\left(\boldsymbol{a}^{\prime}\right)=0$ for $a \leqq b$, and $\hat{\lambda}\left(\boldsymbol{a}^{\prime}\right)=1$ for $a>b$. This implies that $\hat{\lambda}=\hat{\varepsilon}_{b}$. Hence the only idempotent elements of $\mathfrak{P}$ are the measures $\varepsilon_{b}$.

7.4. Definition. Let $\left\{\lambda_{n}\right\}_{n=1}^{\infty}$ be a sequence of measures, where $\lambda_{n} \in \mathfrak{P}$ for all $n$. If there exists $\lambda \in \widetilde{\mathcal{E}}(G)$ such that $\lim _{n \rightarrow \infty} \lambda_{n}(\boldsymbol{x})=\lambda(\boldsymbol{x})$ for all $\boldsymbol{x} \in \hat{G}$, then we say that $\lambda$ is the limit of the sequence $\left\{\lambda_{n}\right\}_{n=1}^{\infty}$, and we write $\lambda=\lim \lambda_{n}$.

7.5. It is easy to show that $\lim \lambda_{n}$ is in $\mathfrak{P}$ whenever it exists. The notion of limit adopted here is very like that employed in the classical theory of probability (see for example [3], pp. 58-62, and esp. 102). There are obvious differences, as we insist on pointwise convergence throughout the entire space of homomorphisms $\hat{G}$, while the classical theory deals only with the homomorphisms defined by integrals $\int_{-\infty}^{\infty} e^{-i x y} d \lambda(x)$, which are not even dense in the space of all homomorphisms (see [12]).

7.6. THEOREM. Let $\lambda \in \mathfrak{F}$ and let $\lambda^{[n]}-\lambda * \cdots * \lambda_{(n)}(n=1,2,3, \cdots)$. Then there is an element $a \in G$ such that $\lim \lambda^{[n]}=\varepsilon_{a}$.

Proof. Consider the function $\hat{\lambda}^{n}=\left(\lambda^{[n]}\right)^{\wedge}$ on the set $\boldsymbol{G}$. Let $A=$ $\{x: x \in G, \hat{\lambda}(\boldsymbol{x})=1\}$. Since $\omega \in A, A$ is non-void. Let $a=\inf A$. Since $\hat{\lambda}$ is non-decreasing, we have $\{x: x \in G, x>a\} \subset A$. Since $\hat{\lambda}$ is continuous on $\boldsymbol{G}$, we have $a \in A$. It follows that

$$
\hat{\lambda}(\boldsymbol{x})\left\{\begin{array}{lll}
=1 & \text { if } \quad x \geq a \\
<1 & \text { if } & x<a
\end{array}\right.
$$


This implies that

$$
\lim _{n \rightarrow \infty} \hat{\lambda}(\boldsymbol{x})=\left\{\begin{array}{lll}
0 & \text { if } & x<a \\
1 & \text { if } & x \geq a
\end{array}\right.
$$

Therefore $\lim _{n \rightarrow \infty} \hat{\lambda}^{n}(\boldsymbol{x})=\hat{\varepsilon}_{a}(\boldsymbol{x})$ for all $\boldsymbol{x} \in \boldsymbol{G}$. The same relation holds if $\boldsymbol{x} \in \boldsymbol{G}^{\prime}$, as is shown by the same argument. (Continuity shows that $a=\inf \left\{x: x \in G, \hat{\lambda}\left(\boldsymbol{x}^{\prime}\right)=1\right\}$.) Therefore, by $7.4, \lim \lambda^{[n]}=\varepsilon_{a}$.

7.7. Finally, we may look for the class of probability measures in $\mathfrak{P}$ that can be written in the form $\lim \left(\mu_{1} * \cdots * \mu_{n}\right)$, where $\mu_{n} \in \mathfrak{P}$. For this purpose, it is convenient to go over to $\hat{\mathfrak{F}}$. Let $\lambda$ be an arbitrary element of $\mathfrak{s}$. Write $\hat{\lambda}=p$. Then for every positive integer $r$, there is a unique nonnegative function $p^{1 / r}$ on $\hat{G}$. It is easy to see that $p^{1 / r}$ satisfies the conditions of 6.7 and has the property that $p^{1 / r}(\boldsymbol{\omega})=1$. Hence $p^{1 / r}$ is the Fourier transform of a probability $\mu_{r}$ such that $u_{r}^{[r]}=\lambda$. It is furthermore clear that

$$
\lim _{n \rightarrow \infty} p^{1 / 2}(\boldsymbol{x}) \cdot p^{1 / 4}(\boldsymbol{x}) \cdot \cdots \cdot p^{1 / 2 n}(\boldsymbol{x})=p(\boldsymbol{x})
$$

for all $\boldsymbol{x} \in \hat{G}$. Therefore, if we write $\lambda_{n}=\mu_{2} * \mu_{4} * \cdots * \mu_{2^{n}}$, we have $\lim \lambda_{n}=$ $\lambda$. Therefore every $\lambda$ in $\mathfrak{F}$ is an "infinite product". If $\lambda$ is not idempotent (that is, not of the form $\varepsilon_{a}$ ), then no $\mu_{r}$ is idempotent, and $\lambda$ is an infinite product with " nondegenerate" factors.

If $\varepsilon_{\alpha}=\lim \left(\mu_{1} * \cdots * \mu_{n}\right)$, then it is clear that all $\mu_{j}$ are equal to $\varepsilon_{\alpha}$. For in the contrary case, we have $\hat{\mu}_{j}(\boldsymbol{a})<1$ for some $\boldsymbol{a} \in \boldsymbol{G}$ and some positive integer $j$. Hence $\lim _{n \rightarrow \infty} \hat{\mu}_{1}(\boldsymbol{a}) \cdots \hat{\mu}_{n}(\boldsymbol{a}) \leqq \hat{\mu}_{j}(\boldsymbol{a})<1$, and $\hat{\mu}_{1} \cdot \cdots \cdot \hat{\mu}_{n}$ does not converge to $\hat{\varepsilon}_{\alpha}=1$ everywhere on $G$.

On the other hand, if $b \in G$ and $b>\alpha$, choose any $u \in G$ such that $\alpha \leqq u<a$. A simple calculation shows that

$$
\lim _{n \rightarrow \infty}\left(\frac{1}{2} \hat{\varepsilon}_{u}(\boldsymbol{x})+\frac{1}{2} \hat{\varepsilon}_{a}(\boldsymbol{x})\right)^{n}=\hat{\varepsilon}_{a}(\boldsymbol{x})
$$

uniformly on $\hat{G}$. Hence $\varepsilon_{a}$ is an infinite product with all factors nondegenerate. (For the case of a finite $G$, see [6], 8.2.)

7.8. An intuitive interpretation of the results of 7.1-7.7 may be given. Consider a game whose possible outcomes are points of $G$, with the probability that the outcome lies in $A \subset G$ given by $\lambda(A)$, where $\lambda \in \mathfrak{P}$. We play the game repeatedly and keep score as follows. After the first game, we take its outcome, $x_{1}$, as our "score". After each subsequent game, we take as our score the maximum of its outcome and our previous score. That is, the score after $n$ games is $\max \left(x_{1}\right.$, 
$\left.\cdots, x_{n}\right)$. The probability that this score lies in $A \subset G$ is $\lambda^{[n]}(A)$. Hence, as $n \rightarrow \infty, 7.6$ shows that the outcome is almost certainly $a$, where $a=\inf \{x: x \in G, \lambda(] x, \omega])=0\}$. This is in accordance with what one intuitively expects. If there is a positive probability of obtaining $x$ in some interval $[a, b]$, then, after sufficiently many repetitions, the probability is arbitrarily close to 1 that the maximum will be greater than or equal to $a$.

A similar interpretation, based on 7.7, can be given for games with different probabilities $\lambda_{n}$. Here an arbitrary $\lambda \in \mathfrak{F}$ can be obtained as the limiting probability as the number of games goes to $\infty$.

\section{Examples and special results.}

Our construction yields interesting results in certain classical cases. We here list a few of them.

8.1. Let $G$ be the closed interval $[0,1]$ on the real line, with the usual ordering. Then $\widetilde{\mathfrak{S}}(G)$ consists of all complex, finite, countably additive Borel measures on $[0,1]$. The space $\hat{G}$ is the union $I \cup I^{\prime} \cup\{1\}$, where $I=\left[0,1\left[\right.\right.$ and $I^{\prime}$ is a replica of $\left.] 0,1\right]$ disjoint from $I$ and $\{1\}$. The point 1 is isolated. Sets of the form $\left[t, t+\delta[\cup] t^{\prime}, t^{\prime}+\delta^{\prime}\right]$, where $[t, t+\delta[$ $\subset I$ and $\left.] t^{\prime}, t^{\prime}+\delta^{\prime}\right] \subset I^{\prime}$, are a basis for open sets in $I \cup I^{\prime}$. This topology was described many years ago by Alexandroff and Urysohn for counterexample purposes [1], and it seems remarkable that it turns up here as the maximal ideal space of a certain Banach algebra.

As noted in 6.9 , the Fourier transforms $\hat{\lambda}$ are just the continuous functions on $G$ that have finite variation on $I \cup\{1\}$. Now let $\varphi$ be any complex-valued function on $[0,1]$ that has finite variation and is continuous on the right: $\varphi(t+0)=\varphi(t)$ for $0 \leqq t<0$. It is well known ([3], p. $53)$ that $\varphi$ determines and is determined by a $\lambda \in \widetilde{\mathfrak{S}}(G): \varphi(t)=\lambda([0, t])$ $(0 \leqq t \leqq 1)$. Hence $\hat{\lambda}(t)=\varphi(t)$ for all $t \in I \cup\{1\}$, and it is easy to see that $\hat{\lambda}\left(t^{\prime}\right)=\varphi(t-0)$ for $t^{\prime} \in I^{\prime}$. It follows that the algebra $\mathfrak{B}$ of all rightcontinuous functions of finite variation on $[0,1]$ with pointwise operations is isomorphic to the algebra of Fourier transforms $\hat{\lambda}$ and hence to $\tilde{\widetilde{S}}(G)$. Furthermore, the homomorphisms of $\mathfrak{B}$ onto $K$ all have the form $\varphi \rightarrow \varphi(t)$ $(0 \leqq t \leqq 1)$ or $\varphi \rightarrow \varphi(t-0) \quad(0<t \leqq 1)$. This answers a question put to the first-named author by Professor Einar Hille in 1946. Finally, if $\varphi_{j} \in \mathfrak{B}$, and $\varphi_{j}$ corresponds to the measure $\lambda_{j} \in \widetilde{\mathfrak{S}}(G)(j=1, \cdots, m)$, then the function $\varphi_{1} \cdots \cdots \varphi_{m}$ corresponds to $\lambda_{1} * \cdots * \lambda_{m}$.

8.2. Let $G$ be any well-ordered set having a greatest element. It 
is obvious that $G$ is compact and hence $\tilde{\widetilde{S}}(G)$ is an algebra of the kind analyzed in the present paper. The measures in $\widetilde{\mathfrak{C}}(G)$ are all uncomplicated. In fact, if $\lambda \in \widetilde{\mathscr{S}}(G)$, there exists a countable subset $\left\{a_{n}\right\}_{n=1}^{\infty}$ of $G$ and a sequence $\left\{z_{n}\right\}_{n=1}^{\infty}$ of complex numbers such that $\sum_{n=1}^{\infty}\left|z_{n}\right|<\infty$ and such that

$$
\lambda=\sum_{n=1}^{\infty} z_{n} \varepsilon_{a_{n}} .
$$

The proof of this depends upon the following fact.

8.2.2. Let $A$ be a well-ordered set with a greatest element and let $\delta$ be a finitely additive, real-valued, non-negative measure on the Borel sets of $A$ such that $\delta(\{p\})=0$ for all $p \in A$ and $\delta$ is inner regular in the sense that $\delta(P)=\sup \{\delta(F): F$ compact, $F \subset P\}$ for all intervals $P=$ $[\alpha, u[\subset A$. Then $\delta=0$.

Proof. We may suppose that $A$ is infinite. Let $\alpha$ be the least element of $A$ and let $\alpha_{+}$be the successor of $\alpha$. Then $\delta\left(\left[\alpha, \alpha_{+}[)=\right.\right.$ $\delta(\{\alpha\})=0$. Suppose that $u \in A$ and that $\delta([\alpha, t[)=0$ for all $t<u$. If $u$ has an immediate predecessor $u_{-}$, then we have

$$
\delta\left(\left[\alpha, u[)=\delta\left(\left[\alpha, u_{-}\left[\bigcup\left\{u_{-}\right\}\right)=\delta\left(\left[\alpha, u_{-}[)+\delta\left(\left\{u_{-}\right\}\right)=0 .\right.\right.\right.\right.\right.\right.
$$

If $u$ has no immediate predecessor, then for every compact set $F \subset[\alpha, u[$, there is a $t<u$ such that $[\alpha, t] \supset F$. There is also a $t^{\prime}$ such that $t<t^{\prime}<u$, and we have $F \subset[\alpha, t] \subset\left[\alpha, t^{\prime}[\right.$. By our inductive hypothesis, we have $\delta([\alpha, t[]=0$. By the regularity of $\delta$, we infer $\delta([\alpha, u[)=$ $\sup \{\delta(F): F$ compact, $F \subset[\alpha, u[)\}=0$. Hence $\delta([\alpha, u[)=0$ for all $u \in A$. Since $\delta(\{\omega\})=0$, it follows that $\delta(A)=0$.

In proving 8.2.1 from 8.2.2, we may clearly suppose that $\lambda$ is nonnegative (use 1.6.5). Let $\left\{a_{n}\right\}_{n=1}^{\infty}$ be the subset of $G$ consisting of all points for which $\lambda$ is positive, and let $z_{n}=\lambda\left(\left\{a_{n}\right\}\right)$. Then $\delta=\lambda-\sum_{n=1}^{\infty} z_{n} \varepsilon_{a_{n}}$ is a measure satisfying the hypothesis of 8.2.2 (this $\delta$ is even countably additive).

It follows that the algebra $\widetilde{\widetilde{S}}(G)$ is isomorphic to the algebra $l_{1}(G)$ described in [8]. Since we have obtained all of the semicharacters of $G$ in the present case, Theorems $1.8,3.3$, and 4.4 of the present paper are somewhat more precise that the corresponding Theorems 5.1, 2.7, and 5.8 of [8].

8.3. As another illustration of our techniques, we find all idempotent elements in $\widetilde{\mathfrak{S}}(G)$, where $G$ satisfies 1.5 . If $\lambda * \lambda=\lambda$, then $\hat{\lambda}^{2}=\hat{\lambda}$ and $\hat{\lambda}$ can 
assume only the values 0 and 1 . According to $6.9, \hat{\lambda}$ must have finite variation on $\boldsymbol{G}$ and be continuous on $\hat{G}$. Hence $\hat{\lambda}$ can have only a finite number of changes of $\operatorname{sign}$ on $\boldsymbol{G}$. A simple argument shows that there exists a finite subset $\left\{b_{j}\right\}_{j=1}^{m}$ of $G$ such that $\alpha<b_{1}<b_{2}<\cdots<b_{m}<\omega$ (we write $\alpha=b_{0}, \omega=b_{m+1}$ in the following formulas) with the following properties. First, we may have

$$
2 \hat{\lambda}(\boldsymbol{x})=\left\{\begin{array}{lll}
1-(-1)^{k} & \text { for } & \boldsymbol{x} \in\left[\boldsymbol{b}_{k}, \boldsymbol{b}_{k+1}[\quad(k=0, \cdots, m),\right. \\
1-(-1)^{m} & \text { for } & \boldsymbol{x} \in\left[\boldsymbol{b}_{m}, \boldsymbol{b}_{m+1}\right]
\end{array}\right.
$$

Second, we may have

$$
2 \hat{\lambda}(\boldsymbol{x})=\left\{\begin{array}{lll}
1+(-1)^{k} & \text { for } & \boldsymbol{x} \in\left[\boldsymbol{b}_{k}, \boldsymbol{b}_{k+1}[\quad(k=0, \cdots, m),\right. \\
1+(-1)^{m} & \text { for } & \boldsymbol{x} \in\left[\boldsymbol{b}_{m}, \boldsymbol{b}_{m+1}\right] .
\end{array}\right.
$$

These are the only possibilities. Translating this into a statement about the original measures, we see that $\lambda$ must have the form

$$
\lambda=\varepsilon_{c_{0}}-\varepsilon_{c_{1}}+\varepsilon_{c_{2}}+\cdots+(-1)^{k} \varepsilon_{c_{k}},
$$

where $\alpha \leqq c_{0}<c_{1}<\cdots<c_{k} \leqq \omega$. Since every measure 8.3.3 is obviously idempotent, we have found all idempotent measures in $\widetilde{5}(G)$. This may be compared with Theorem 9.1 of [8], where we obtain a less precise result for a class of measure algebras related to but more complicated than those under study here.

8.4. Again let $G$ satisfy 1.5. $\widetilde{\subseteq}(G)$ admits an obvious involution. Let $L \in \hat{\mathcal{S}}(G)$ and $L=M+i N$, where the functionals $M$ and $N$ are realvalued for real-valued $f \in \mathfrak{S}(G)$. Then the mapping $L \rightarrow \overline{\bar{L}}=M-i N$ is an involution of $\widetilde{\mathfrak{S}}(G)$. Furthermore, $\widetilde{\mathfrak{S}}(G)$ is obviously symmetric under this involution: $(\overline{\bar{\lambda}})^{\wedge}$ is the complex conjugate of $\hat{\lambda}$. However, $\widetilde{\mathfrak{S}}(G)$ is never isomorphic to $\tilde{\mathfrak{S}}(G)$ (pointwise operations) if $G$ is infinite. If $G$ is infinite, we may suppose without loss of generality that $G$ contains an infinite strictly increasing subset

$$
a_{1}<a_{2}<a_{3}<\cdots<a_{n}<\cdots .
$$

Let $b$ be the least upper bound of this set. It is easy to see that $T=\left\{\boldsymbol{a}_{n}\right\} \cup\left\{\boldsymbol{b}^{\prime}\right\}$ is a closed subset of $\hat{G}$. The function $\gamma$ on $T$ such that $\gamma\left(\boldsymbol{a}_{n}\right)=\frac{1}{n}\left(1-(-1)^{n}\right)$ and $\gamma\left(\boldsymbol{b}^{\prime}\right)=0$ is continuous on $T$. By Tietze's extension theorem, there is a continuous function $\gamma_{0}$ on $\hat{G}$ such that $\gamma_{0}\left(\boldsymbol{a}_{n}\right)=$ $\gamma\left(\boldsymbol{a}_{n}\right)$ ([9], p. 242). Obviously $\gamma_{0}$ has infinite variation on $\boldsymbol{G}$ and hence is not a Fourier transform (6.9). 
8.5. Following a suggestion of the referee, we note that if a semigroup $G$ satisfies all of the hypotheses of 1.1-1.3 and if 1.4 is replaced by the hypothesis of local compactness, then it can be treated in much the same way as we have treated the compact case. Certain changes, however, are needed. The function space $\sqrt{5}(G)$ of 1.6 is replaced by ${ }^{*} *(G)$, the space of all bounded continuous functions on $G$. The conjugate space $\widetilde{\mathfrak{S}}(G)$ is replaced by $\mathscr{C}(G)$, the space of all countably additive, complex-valued, finite Borel measures on $G$. (This is a realization of $\tilde{\mathfrak{S}}(G)$ for $G$ compact but is ordinarily only a very small part of the conjugate space of $\mathcal{C}^{*}(G)$ if $G$ is non-compact.) The integral 1.6.1 exists for all $f \in \mathbb{C}^{*}(G)$ and $\lambda \in \mathscr{C l}(G)$ and defines a bounded linear functional on $C^{*}(G)$. Under this definition, $\mathscr{C l}(G)$ is a convolution algebra. Every semicharacter of $G$ is defined by a Dedekind cut, and it will be of the form $1.8 .1,1.8 .2$, or as in 1.11. $\mathscr{C}(G)$ has a unit if and only if $G$ has a least element $a$ and the unit in this case is $\varepsilon_{\alpha}$. (See 2.10.) The results of $\S \S 3$ and 4 can be carried over with obvious modifications. The maximal ideal space of $\mathscr{L}(G)$ is still $\hat{G}$ (see $\S 5$ ), but the topological structure may be complicated. We omit the details. The changes necessary in $\S \S 6-7$ are considerably greater, and the more general results to be obtained would not seem to justify carrying out all of the details.

\section{REFERENCES}

1. P. S. Alexandroff and Paul Urysohn, Mémoire sur les espaces compacts, Verh. Neder1. Akad. Wetensch. Afd. Natuurk., 14, No. 1.

2. N. Bourbaki, Éléments de mathématique, Première partie, Livre VI. Intégration, Ch. I-V. Act. Sci. et Ind. 1175. Hermann et Cie., Paris, 1952.

3. Harald Cramér, Mathematical methods of statistics, Princeton University Press, 1946.

4. Paul Halmos, Measure theory, D. Van Nostrand Co., Inc., New York, 1950.

5. Edwin Hewitt, Remarks on the inversion of Fourier-Stieltjes transforms, Ann. of Math. (2 Ser.) 57 (1953), 458-474.

6. Edwin Hewitt and Herbert S. Zuckerman, Arithmetic and limit theorems for a class of random variables, Duke Math. J., 22 (1955), 595-615.

7. - Finite dimensional convolution algebras, Acta Math. 93 (1955), 67-119.

8. The $l_{1}$ algebra of a commutative semigroup, Trans. Amer. Math. Soc. 83 (1956), 70-97.

9. J. L. Kelley, General topology. New York, D. Van Nostrand Co., Inc., 1955.

10. Lynn H. Loomis, An introduction to abstract harmonic analysis, D. Van Nostrand Co., Inc., New York, 1953.

11. I. P. Natanson, Theory of functions of a real variable, Frederick Ungar, New York, 1955.

12. Yu. A. Šreŭder, Stroenie maksimal'nyh idealov $v$ kol'cah mer so svertkoŭ, Mat. Sb. N.S. 27 (69), (1950), 297-318. 



\section{PACIFIC JOURNAL OF MATHEMATICS}

EDITORS

H. L. ROYDEN

Stanford University

Stanford, California

R. A. Beaumont

University of Washington

Seattle 5 , Washington
A. R. Whiteman

University of Southern California

Los Angeles 7, California

E. G. Straus

University of California

Los Angeles 24, California

\section{ASSOCIATE EDITORS}
E. F. BECKENBACH
C. E. BURGESS
M. HALL
E. HEWITT
A. HORN
V. GANAPATHY IYER
R. D. JAMES
M. S. KNEBELMAN

L. NACHBIN

I. NIVEN

G. SZEKERES

T. G. OSTROM

M. M. SCHIFFER
F. WOLF

K. YOSIDA

\section{SUPPORTING INSTITUTIONS}

UNIVERSITY OF BRITISH COLUMBIA

CALIFORNIA INSTITUTE OF TECHNOLOGY

UNIVERSITY OF CALIFORNIA

MONTANA STATE UNIVERSITY

UNIVERSITY OF NEVADA

OREGON STATE COLLEGE

UNIVERSITY OF OREGON

UNIVERSITY OF SOUTHERN CALIFORNIA

\author{
STANFORD UNIVERSITY \\ UNIVERSITY OF UTAH \\ WASHINGTON STATE COLLEGE \\ UNIVERSITY OF WASHINGTON \\ AMERICAN MATHEMATICAL SOCIETY \\ CALIFORNIA RESEARCH CORPORATION \\ HUGHES AIRCRAFT COMPANY \\ THE RAMO-WOOLDRIDGE CORPORATION
}




\section{Pacific Journal of Mathematics}

\section{Vol. 7, No. $1 \quad$ January, 1957}

Richard Davis Anderson, Zero-dimensional compact groups of

homeomorphisms ................................... 797

Hans-Joachim Bremermann, Holomorphic functionals and complex

convexity in Banach spaces........................... 811

Hugh D. Brunk, G. M. Ewing and W. R. Utz, Minimizing integrals in

certain classes of monotone functions ................. 833

Philip David, Uniqueness theory for asymptotic expansions in general

regions ...................................... 849

Paul Erdős and Harold Nathaniel Shapiro, On the least primitive root of a

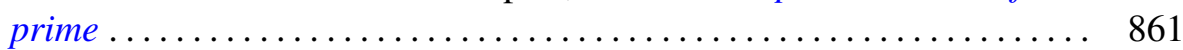

Watson Bryan Fulks, Regular regions for the heat equation ........... 867

William Robert Gaffey, A real inversion formula for a class of bilateral

Laplace transforms ................................ 879

Ronald Kay Getoor, On characteristic functions of Banach space valued random variables ................................. 885

Louis Guttman, Some inequalities between latent roots and minimax (maximin) elements of real matrices ...................... 897

Frank Harary, The number of dissimilar supergraphs of a linear graph .... 903

Edwin Hewitt and Herbert S. Zuckerman, Structure theory for a class of convolution algebras .................................. 913

Amnon Jakimovski, Some Tauberian theorems . . . . . . . . . . . . . . . . . 943

C. T. Rajagopal, Simplified proofs of "Some Tauberian theorems" of Jakimovski................................

Paul Joseph Kelly, A congruence theorem for trees ................. 961

Robert Forbes McNaughton, Jr., On the measure of normal formulas...... 969

Richard Scott Pierce, Distributivity in Boolean algebras .............. 983

Calvin R. Putnam, Continuous spectra and unitary equivalence ......... 993

Marvin Rosenblum, Perturbation of the continuous spectrum and unitary

equivalence................................... 997

V. N. Singh, Certain generalized hypergeometric identities of the

Rogers-Ramanujan type.......................

Peter Swerling, Families of transformations in the function spaces $H^{p} \ldots \ldots 1015$ 